\title{
Elucidation of Cryptic and Allosteric Pockets within the SARS-CoV-2 Main Protease
}

\author{
Terra Sztain ${ }^{1 *}$, Rommie Amaro ${ }^{1}$, J. Andrew McCammon ${ }^{1,2}$ \\ ${ }^{1}$ Department of Chemistry and Biochemistry, University of California, San Diego, La Jolla, California 92093, \\ United States \\ ${ }^{2}$ Department of Pharmacology, University of California, San Diego, La Jolla, California 92093, United States \\ *Correspondence may be addressed to: tsztainp@ucsd.edu
}

\section{Supplementary Figures}

Figures S1 - S26

\section{Supplementary Movie}

Movie S1 Active site pocket dynamics sampled with GaMD

\section{Supplementary Data}

Data S1 Values for RMSD of 275 of $\mathrm{M}^{\text {pro }}$ crystal structures compared to PDB 6LU7 


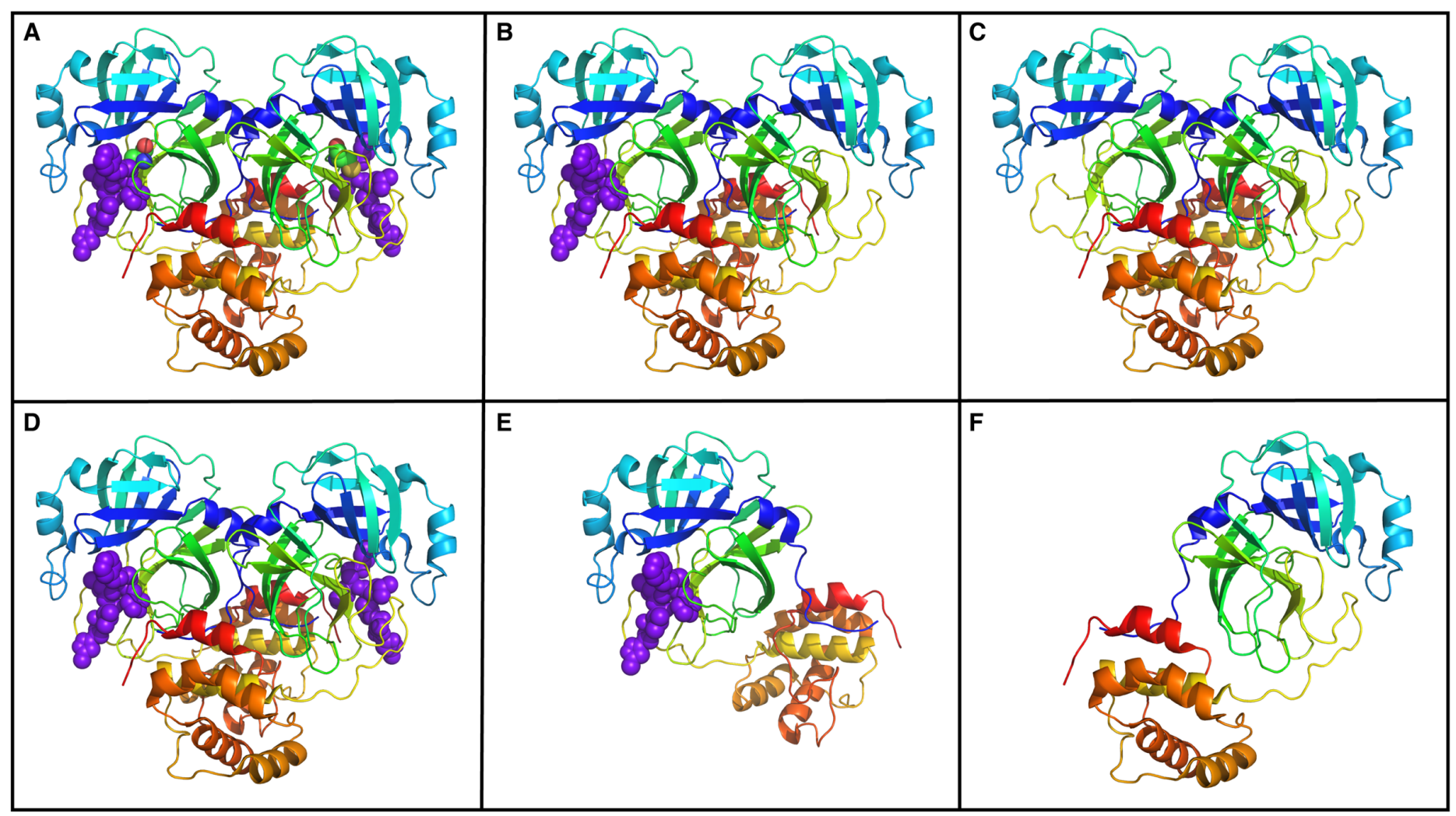

Figure S1 Summary of systems simulated from PDB 6LU7. (A) $M^{\text {pro }}$ dimer with two N3 inhibitor molecules covalently attached to active site cysteine 145 . (B) $M^{\text {pro }}$ dimer with one N3 inhibitor non-covalently bound. (C) $\mathrm{M}^{\text {pro }}$ apo dimer. (D) $\mathrm{M}^{\text {pro }}$ dimer with two N3 inhibitors non-covalently bound. (E) $\mathrm{M}^{\text {pro }}$ monomer with one N3 inhibitor non-covalently bound. (F) $\mathrm{M}^{\text {pro }}$ apo monomer. 
A

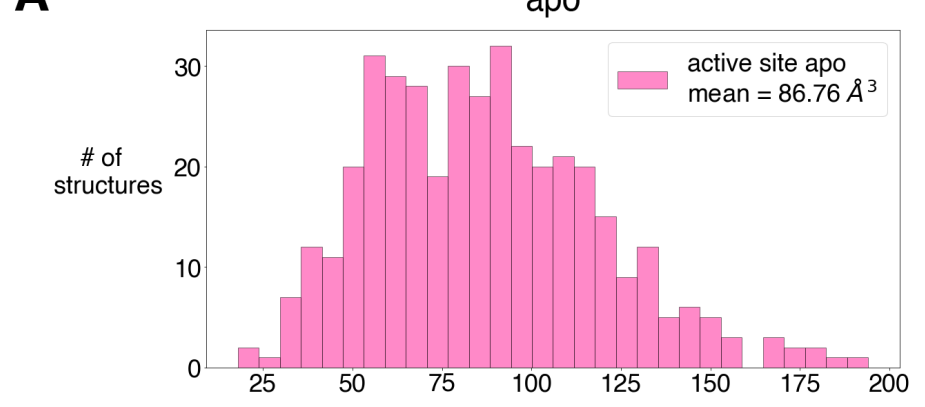

C

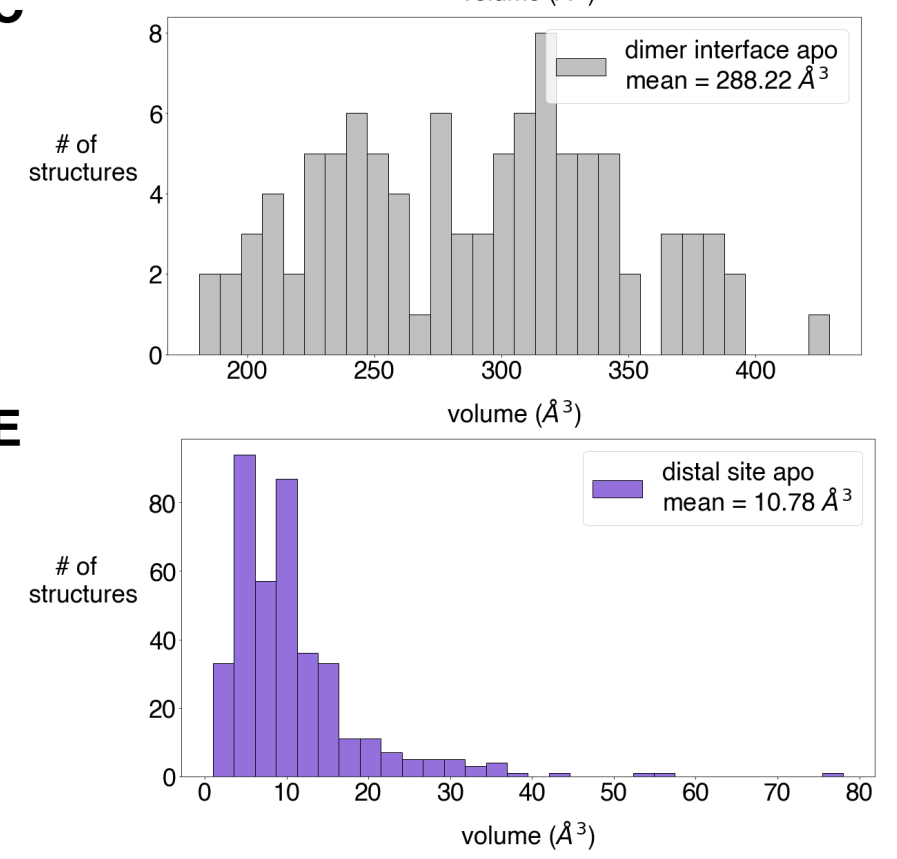

B

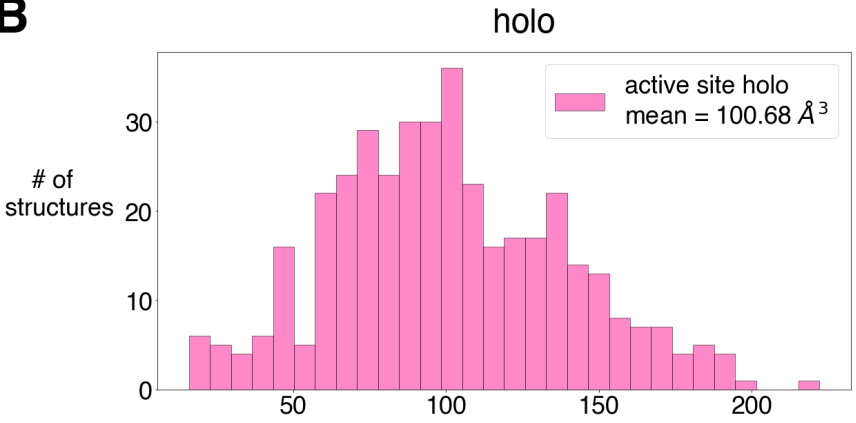

D

volume $\left(\AA^{3}\right)$

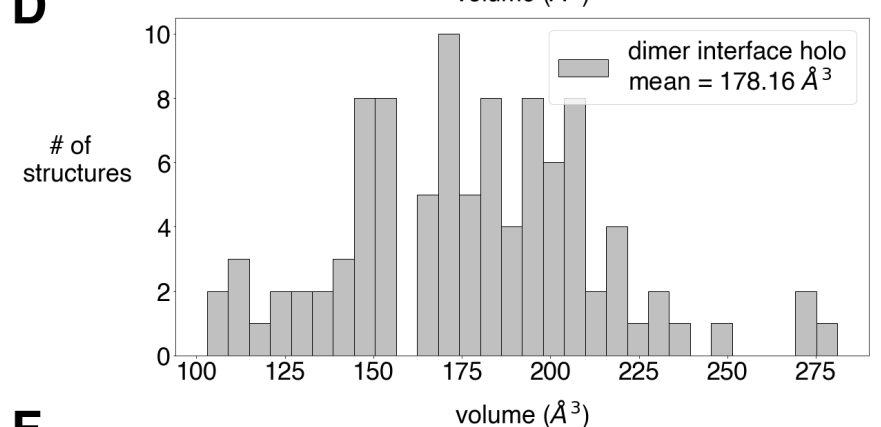

$\mathbf{F}$

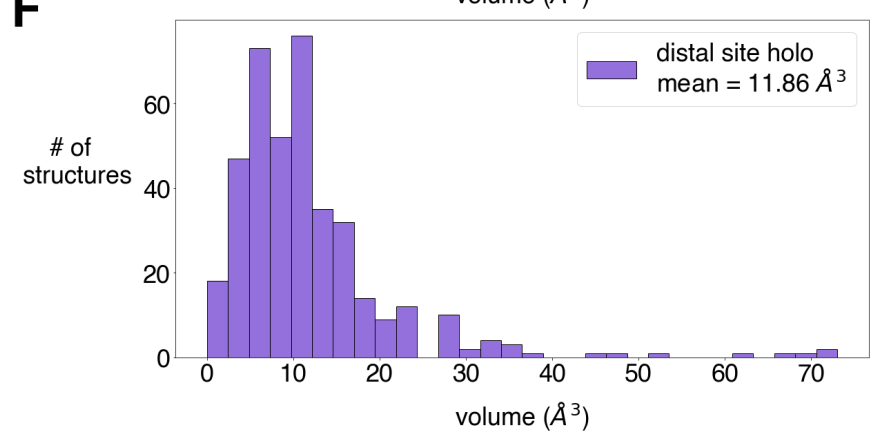

Figure S2 Pocket volume of each region in apo versus N3 bound simulations. Histograms show average pocket volume calculated from aggregate $1 \mu \mathrm{s}$ dimer and monomer simulations of $\mathrm{M}^{\text {pro }}$ in the apo (A,C,E) and holo, N3 bound state $(\mathrm{B}, \mathrm{D}, \mathrm{F})$. Both covalent and non-covalent N3 were included in the definition of holo. The dimer simulation with one N3 bound and one apo active site was omitted from either definition. 

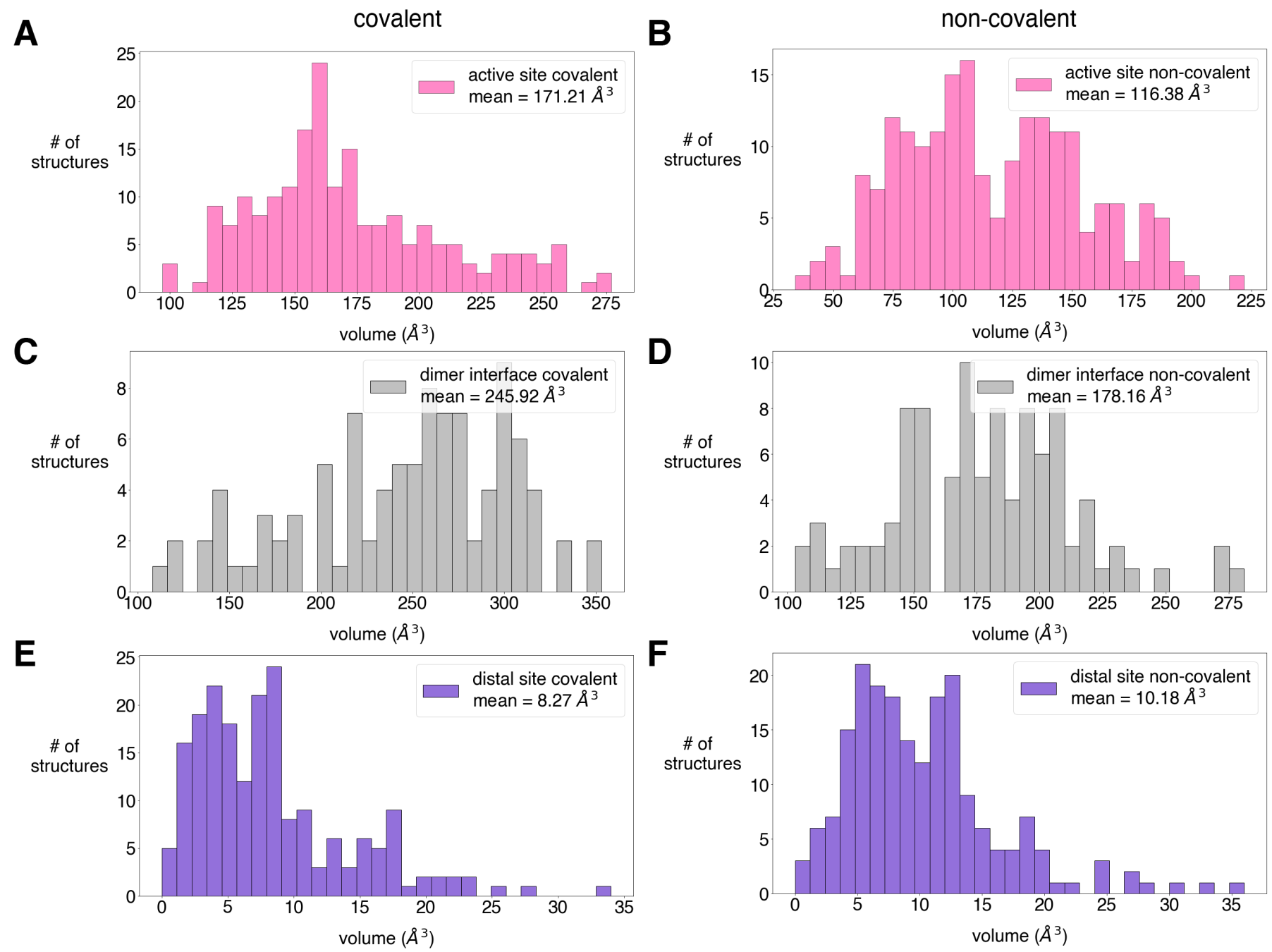

Figure S3 Pocket volume of each region in covalent versus non-covalent simulations. Histograms show average pocket volume calculated from aggregate $1 \mu$ s dimer simulations of $\mathrm{M}^{\text {pro }}$ with two $\mathrm{N} 3$ covalently $(\mathrm{A}, \mathrm{C}, \mathrm{E})$ or non-covalently $(\mathrm{B}, \mathrm{D}, \mathrm{F})$ bound. 

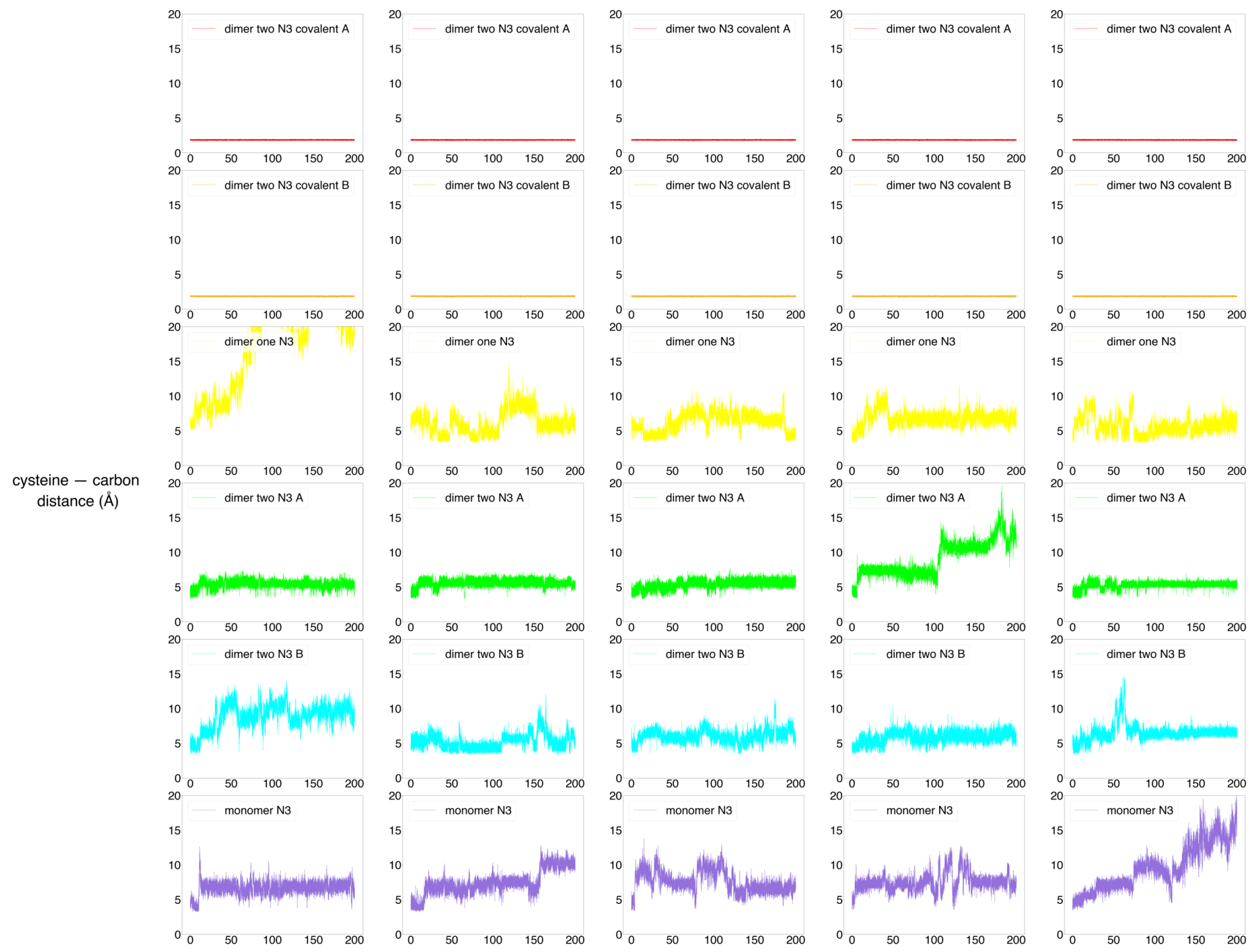

15

10

5

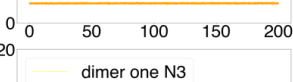

15

5

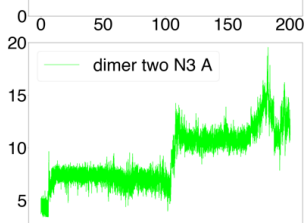

$\begin{array}{lllll}0 & 50 & 100 & 150 & 200\end{array}$

dimer two N3 A

15

10
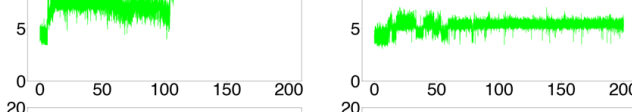

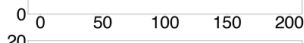

20 dimer two N3 B

15

10

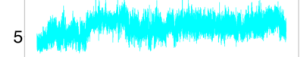

5
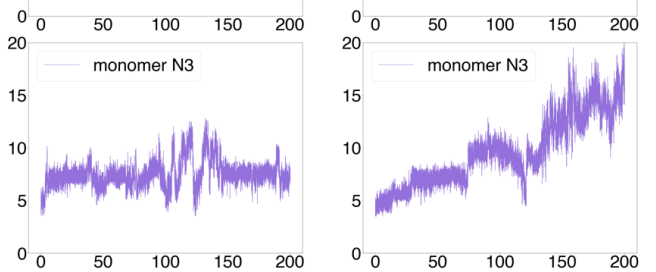

time (ns)

Figure S4 Distance between active site cysteine of $\mathrm{M}^{\text {pro }}$ and carbon of N3 inhibitor which is covalently attached. N3 was simulated non-covalently to $\mathrm{M}^{\text {pro }}$ unless labeled covalent. 



550

500

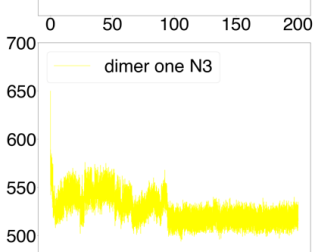

\begin{tabular}{lllll|}
700 & $50 \quad 100 \quad 150 \quad 200$ \\
650 & & dimer two N3 A & \\
& & &
\end{tabular}
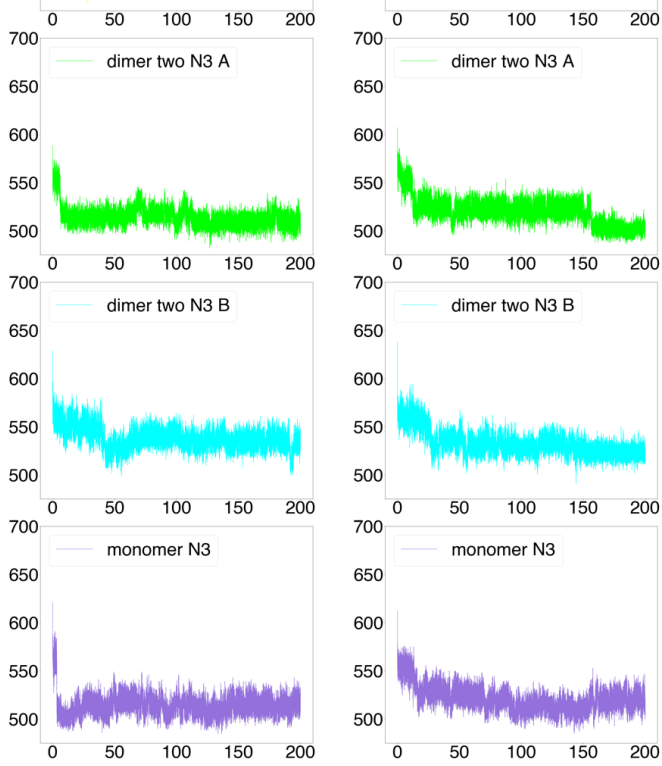

Figure S5 Native contacts of N3 inhibitor throughout simulations. N3 was simulated non-covalently to $\mathrm{M}^{\text {pro }}$ unless labeled covalent. 

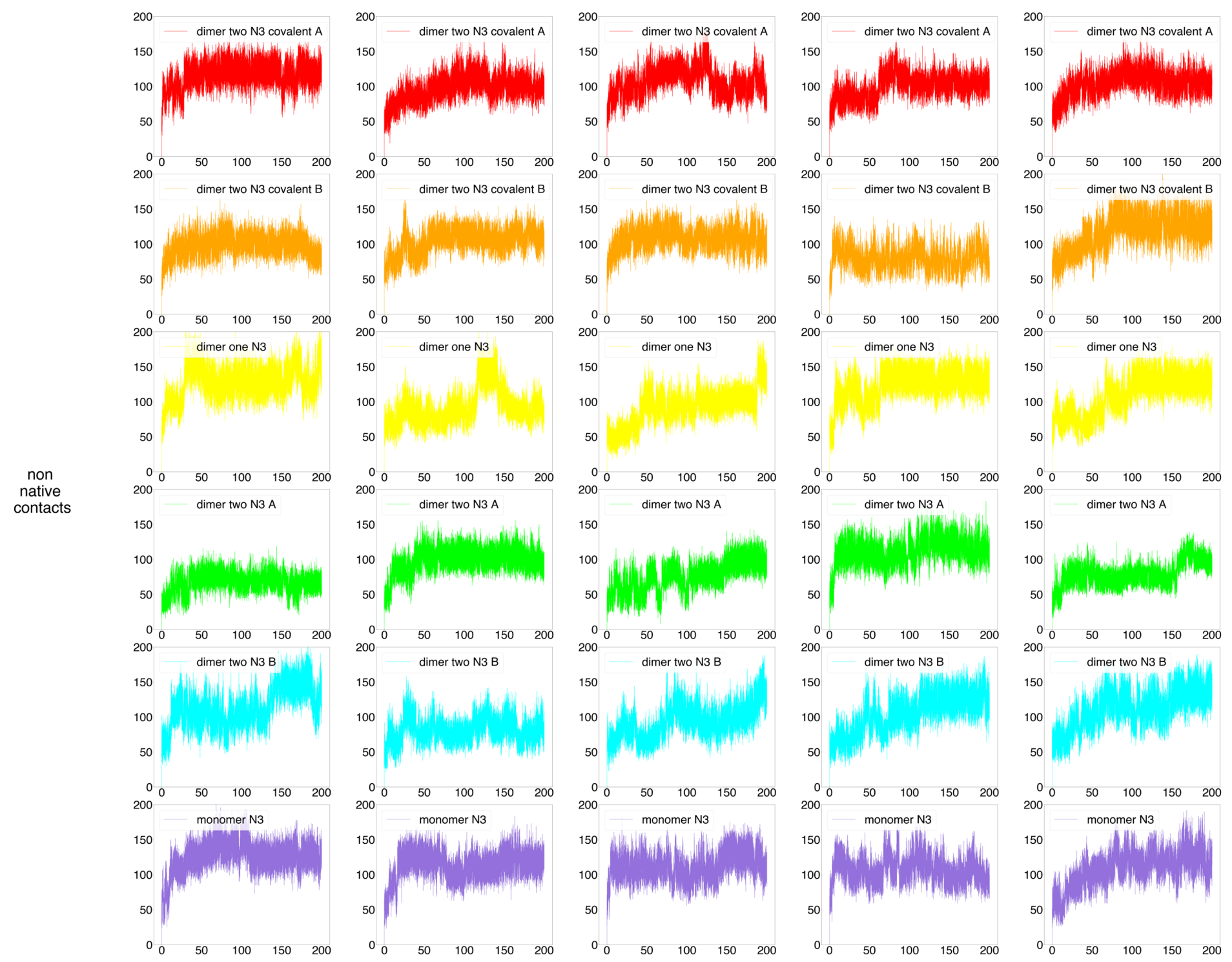

150

150

${ }_{200}^{0} 0 \quad 50 \quad 100 \quad 150 \quad 200$

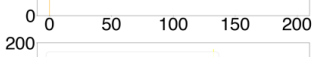

\begin{tabular}{|l|l|l|}
\hline 100 & 100 \\
\hline
\end{tabular}

50

100
50

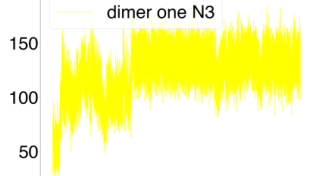

200 dimer one N3

$\begin{array}{lllll}{ }^{0} 0 & 50 & 100 & 150 & 200\end{array}$

$\begin{array}{ccccc}0^{0} 0 & 50 \quad 100 \quad 150 \quad 200 \\ & & \text { dimer two N3 A } & \end{array}$

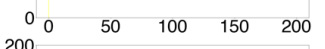

150

100

50

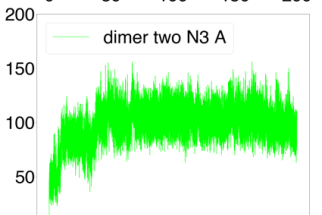

150
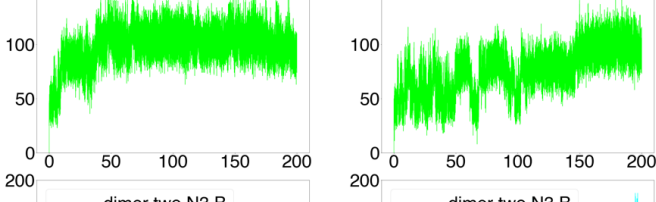

$\begin{array}{rrrrr}{ }^{0} 0 & 50 & 100 & 150 & 200\end{array}$
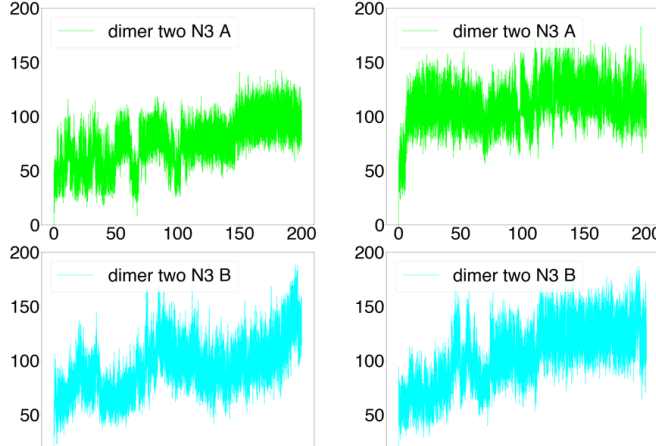

$\begin{array}{lllll}{ }^{0} 0 & 50 & 100 & 150 & 200\end{array}$

200

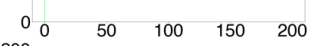

200 dimer two N3 B



150

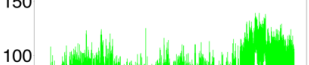

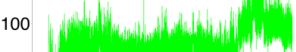

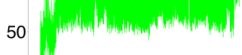

${ }_{0}^{0} 0 \quad 50 \quad 100 \quad 150 \quad 200$

200 dimer two N3 B

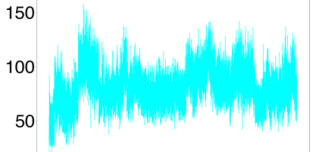

$\begin{array}{lllll}{ }^{0} 0 & 50 & 100 & 150 & 200\end{array}$

$\begin{array}{lllll}0 & 50 & 50 & 150 & 200\end{array}$

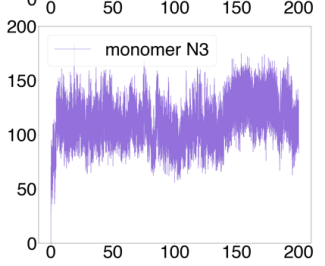

$\begin{array}{lllll}{ }^{0} 0 & 50 & 100 & 150 & 200\end{array}$

150
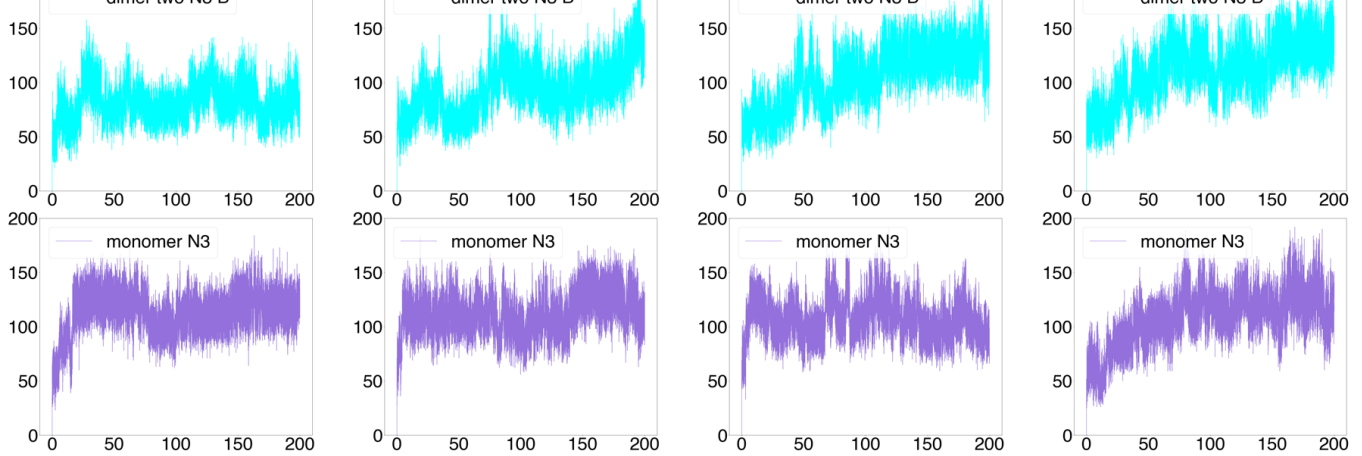

time (ns)

Figure S6 Non-native contacts of N3 inhibitor throughout simulations. N3 was simulated non-covalently to $\mathrm{M}^{\mathrm{pro}}$ unless labeled covalent. 
A

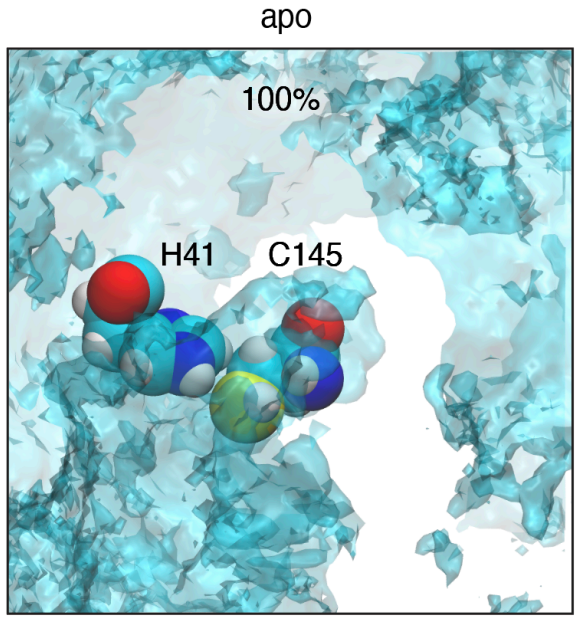

B

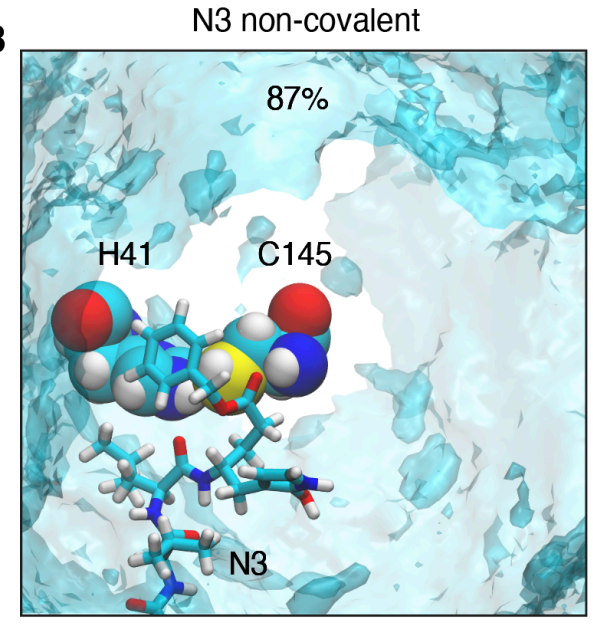

C

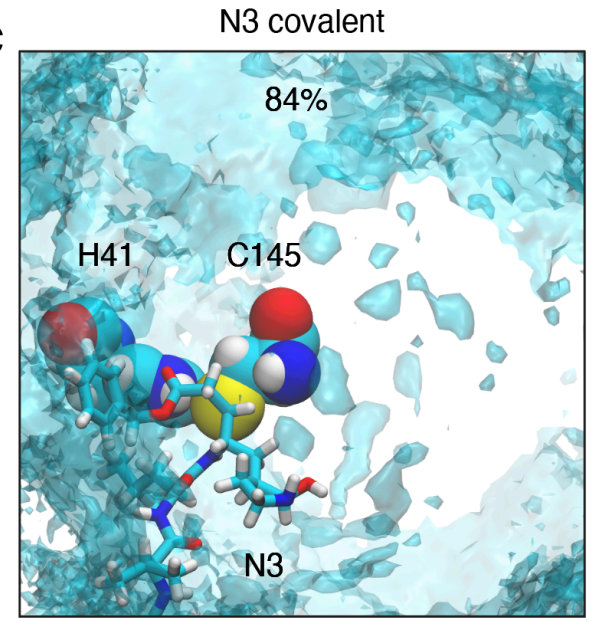

Figure S7 Analysis of water occupancy in the active site. Average water occupancy calculated from $200 \mathrm{~ns}$ GaMD simulation of the (A) apo, (B) N3 non-covalent and (C) N3 covalent simulations. Catalytic dyad residues $\mathrm{H} 41$ and C145 are shown as spheres, and N3 inhibitor, if present, is shown as licorice. Average \% water occupancy compared to apo simulation is labeled. 
1

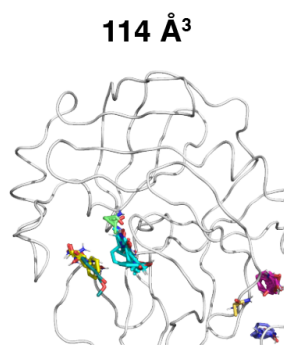

0.65

6

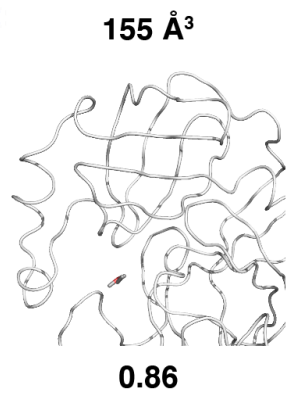

2

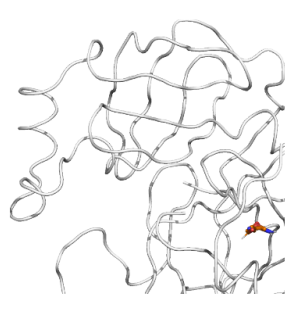

0.65

7

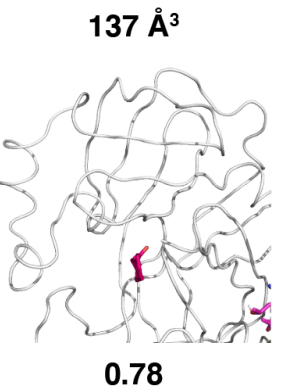

active site pocket

3

$39 \AA^{3}$

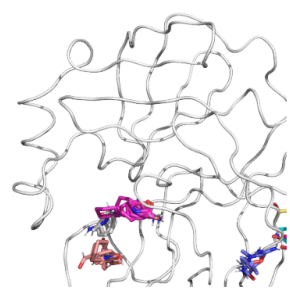

0.56

8

$60 \AA^{3}$

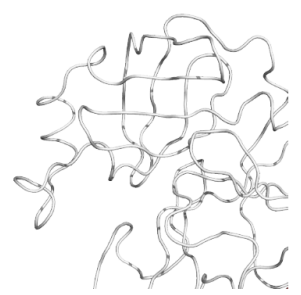

0.67
4

$248 \AA^{3}$

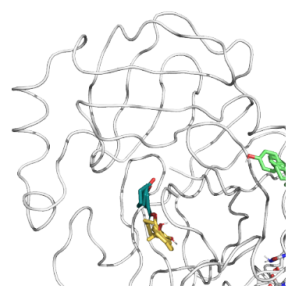

0.18

9

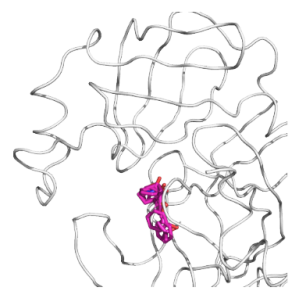

0.65
$5 \quad 91 \AA^{3}$

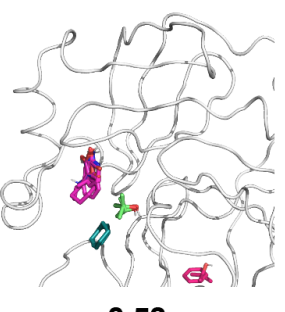

0.53

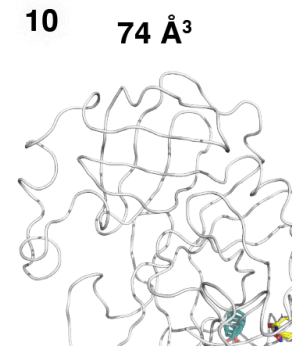

0.35

Figure S8 Druggability and hot-spot mapping of selected active site pockets. POVME ${ }^{13}$ calculated pocket volumes are labeled, along with PockDrug ${ }^{14}$ predicted druggability probability. Solvent molecules predicted to bind with FTMap ${ }^{15}$ are shown as sticks. 


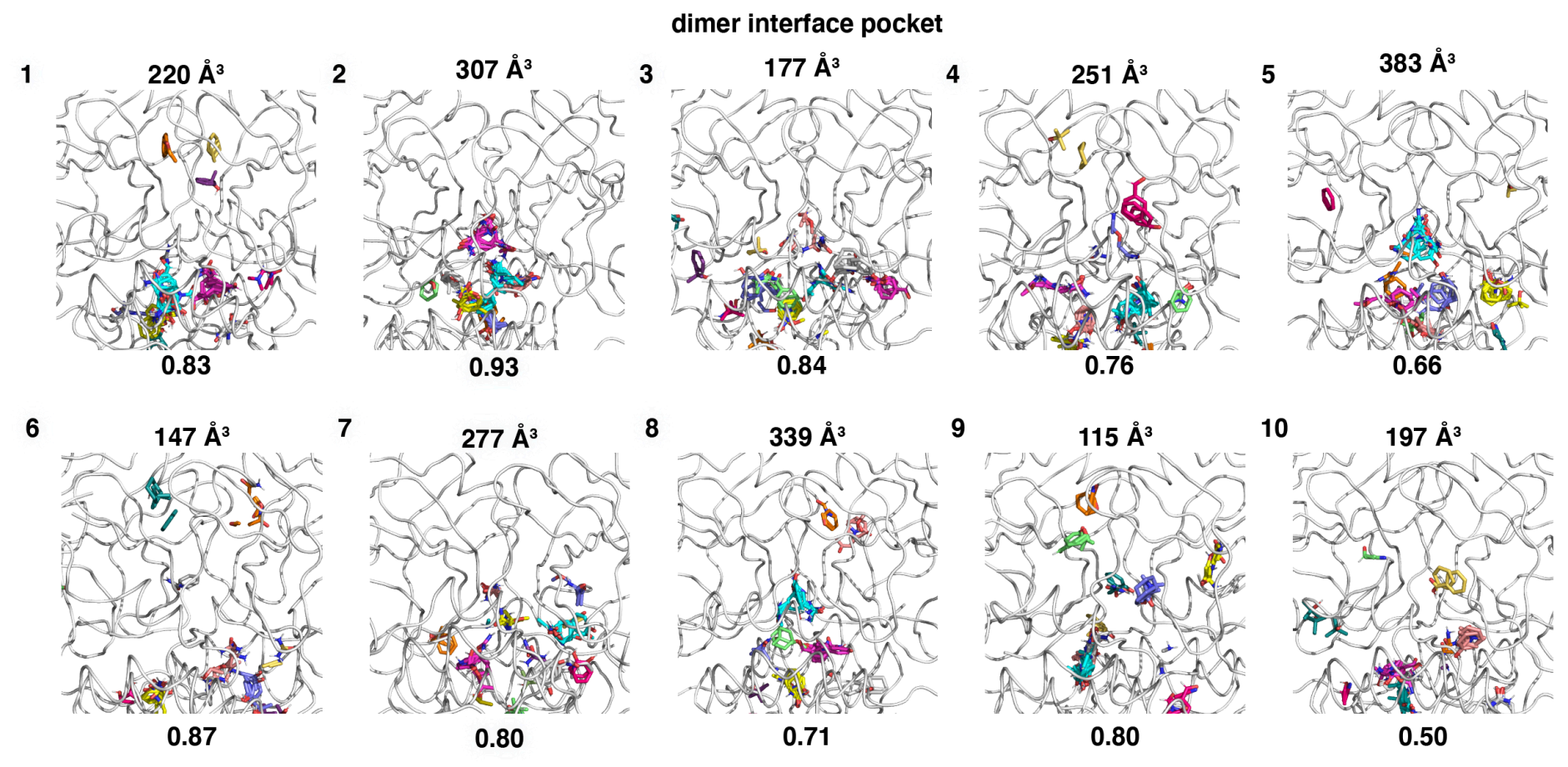

Figure S9 Druggability and hot-spot mapping of selected dimer interface pockets. POVME ${ }^{13}$ calculated pocket volumes are labeled, along with PockDrug ${ }^{14}$ predicted druggability probability. Solvent molecules predicted to bind with FTMap ${ }^{15}$ are shown as sticks. 
distal site pocket

1

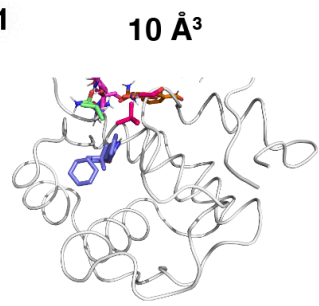

1.00

6

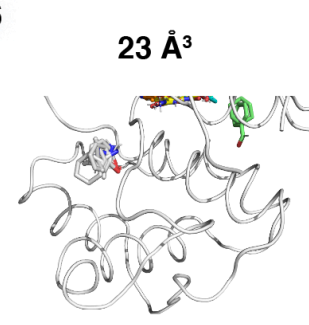

1.00
$213 \AA^{3}$

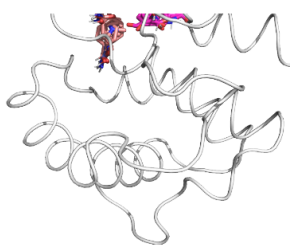

0.94

7

$47 \AA^{3}$

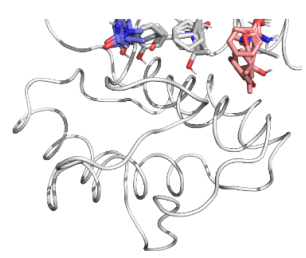

0.82
3

$32 \AA^{3}$

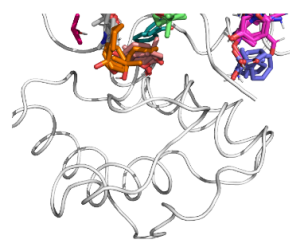

1.00

8

$17 \AA^{3}$

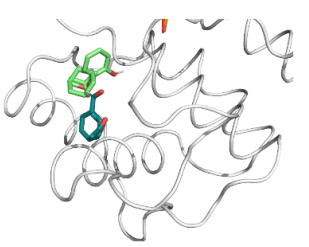

0.37
4

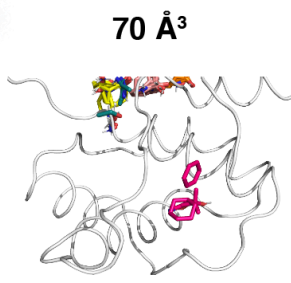

0.94

9

$7 \AA^{3}$

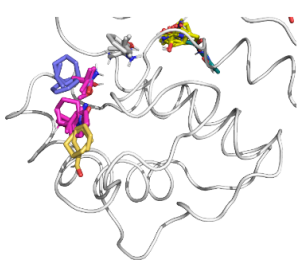

0.78
$5 \quad 4 \AA^{3}$

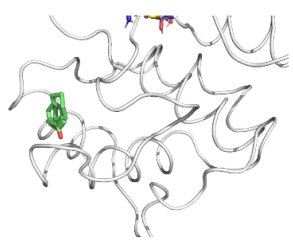

0.98

10
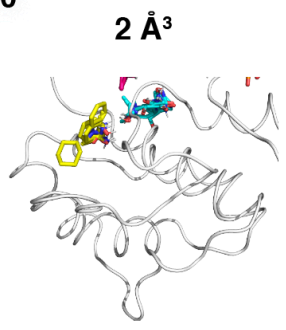

0.76

Figure S10 Druggability and hot-spot mapping of selected distal site pockets. POVME ${ }^{13}$ calculated pocket volumes are labeled, along with PockDrug ${ }^{14}$ predicted druggability probability. Solvent molecules predicted to bind with FTMap ${ }^{15}$ are shown as sticks. 


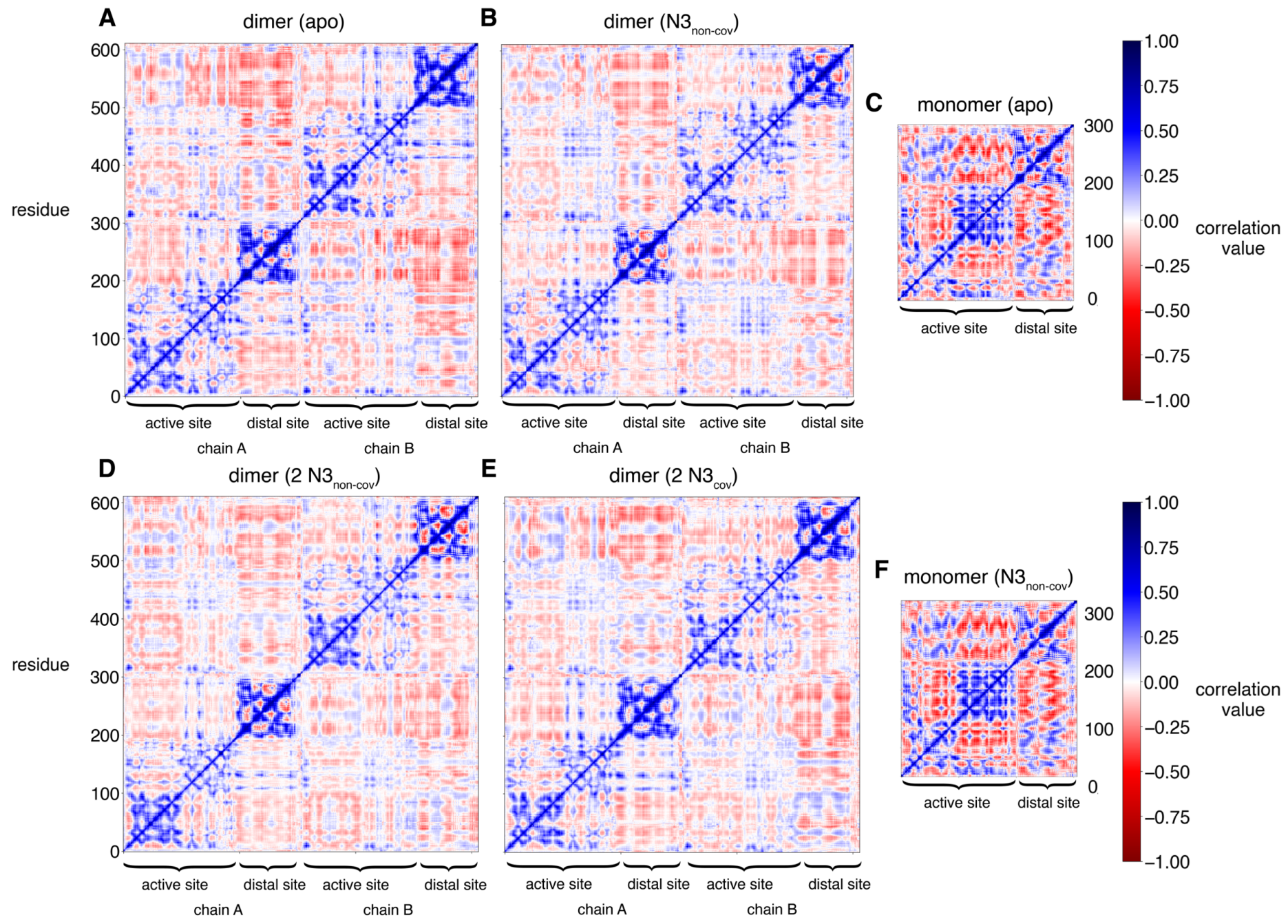

Figure S11 Correlation matrix calculated from aggregate $1 \mu$ s of each simulation. (A) Apo $M^{\text {pro }}$ dimer (B) $M^{\text {pro }}$ dimer with one N3 non-covalently bound. (C) Apo $\mathrm{M}^{\text {pro }}$ monomer. (D) $\mathrm{M}^{\text {pro }}$ dimer with two N3 non-covalently bound. (E) $\mathrm{M}^{\text {pro }}$ dimer with two N3 covalently bound. (F) $M^{\text {pro }}$ monomer with N3 bound. 
A

dimer (apo)

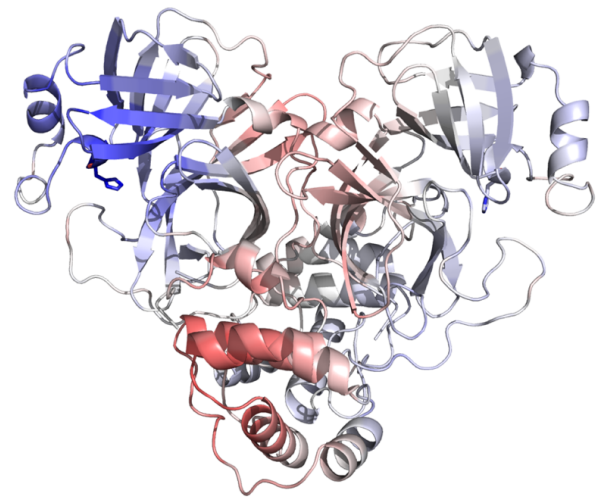

D

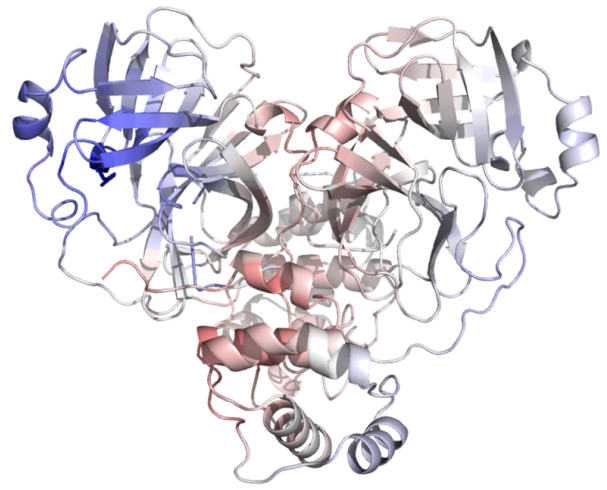

B

$\operatorname{dimer}\left(\mathrm{N} 3_{\text {non-cov }}\right)$

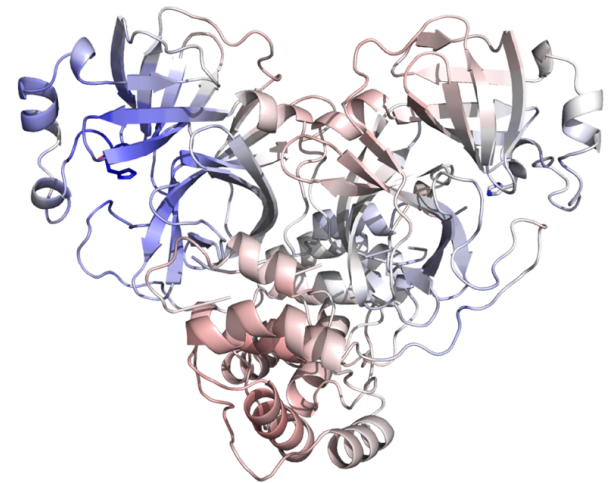

E

$\operatorname{dimer}\left(2 \mathrm{~N}_{\text {cov }}\right)$

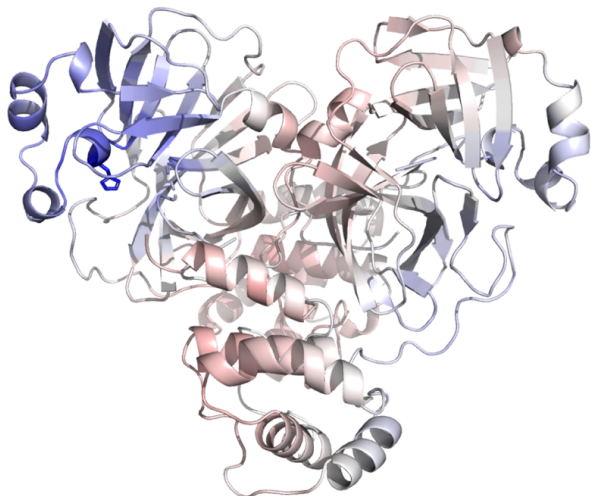

C

monomer (apo)

Figure S12 Structural representation of correlated motions to the catalytic histidine. Each residue is colored based on correlation value to H41, similar to the color scale in Figure S4, for (A) Apo M ${ }^{\text {pro }}$ dimer, (B) M ${ }^{\text {pro }}$ dimer with one non-covalent N3, (C) Apo $\mathrm{M}^{\text {pro }}$ monomer, (D) $\mathrm{M}^{\text {pro }}$ dimer with two non-covalent N3, (E) M ${ }^{\text {pro }}$ dimer with two covalent N3and (F) $\mathrm{M}^{\text {pro }}$ monomer with non-covalent N3. 
A

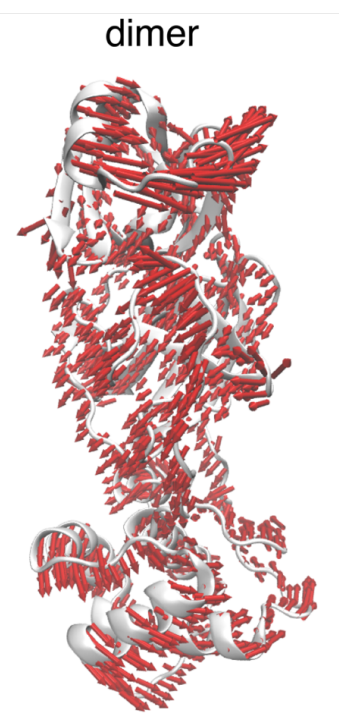

B

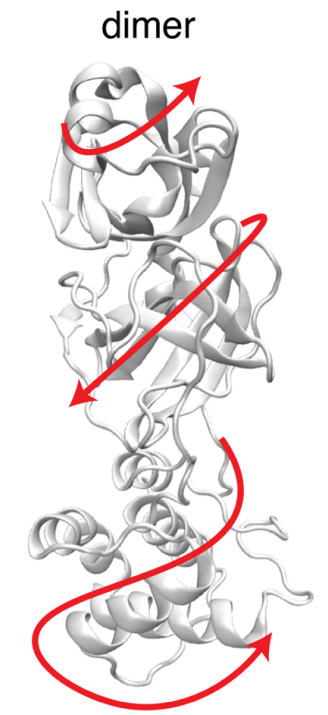

C

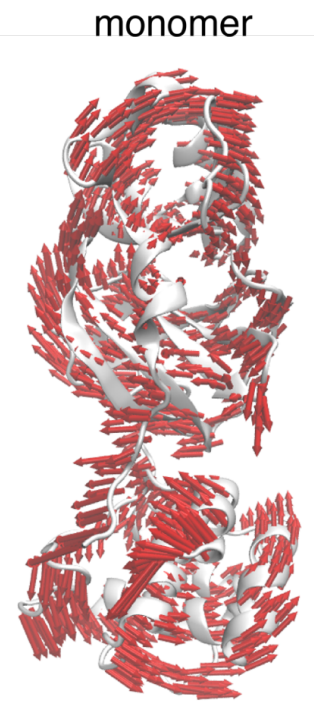

D

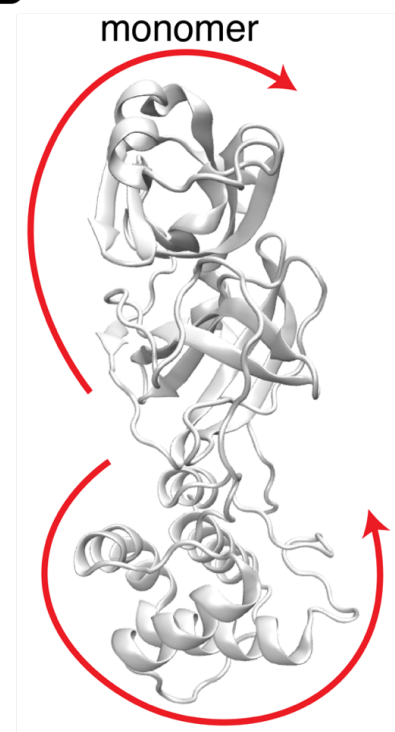

Figure S13 Principal motions during dimer and monomer simulations. The normal modes from the first principal component were projected onto one monomer from the dimer simulation (A) and monomer simulation (C) using VMD. The resulting overall "twisting" motion observed in the dimer is schematically shown by the red arrows in (B) and the "hinging" motion observed in the monomer is shown in (D). 
A

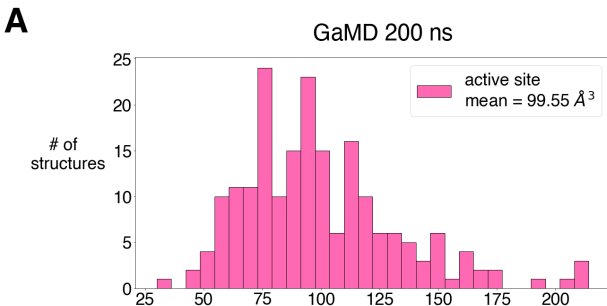

D

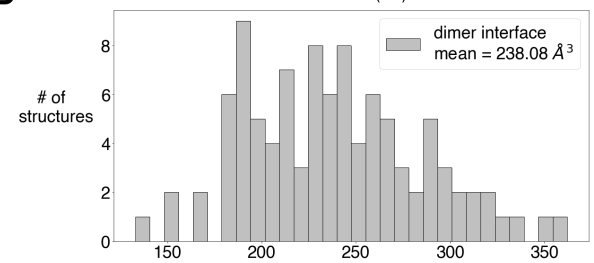

G

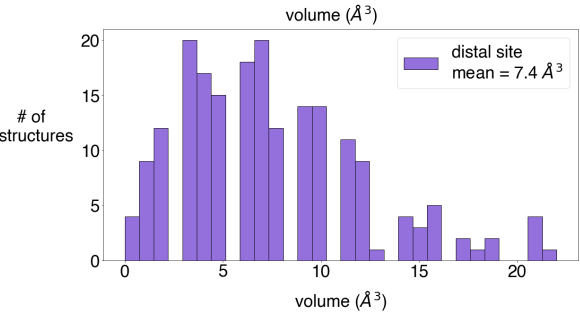

J
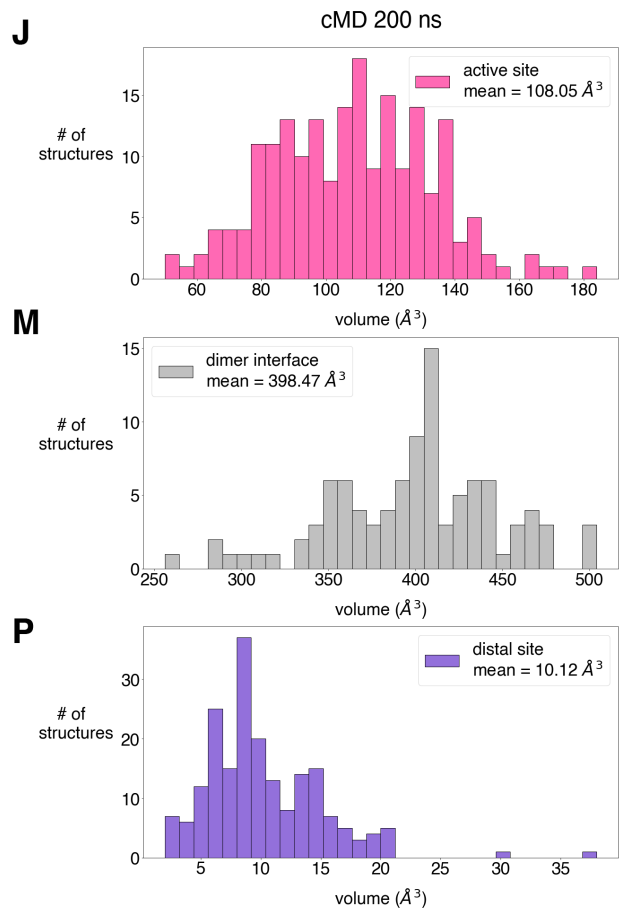

B

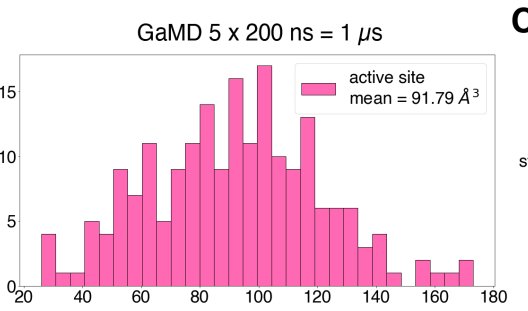

E
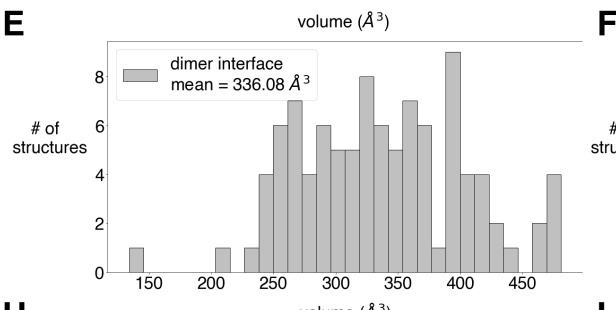

H

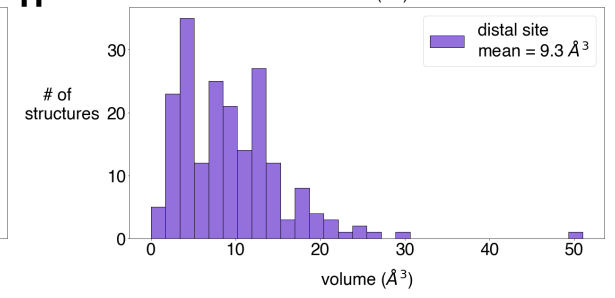

K

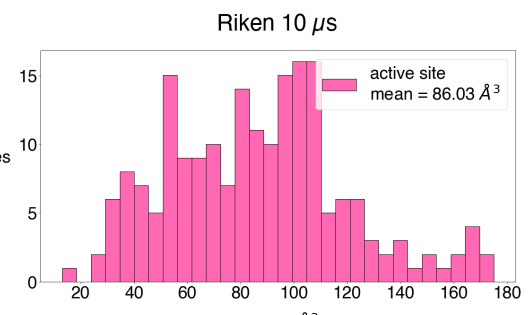

N
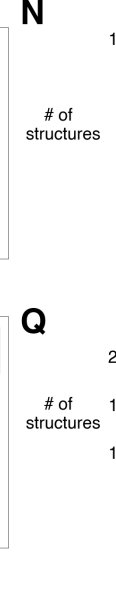

C

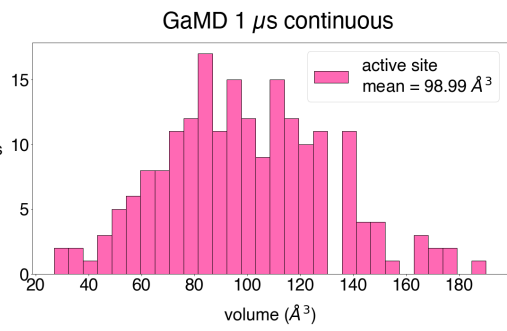

F
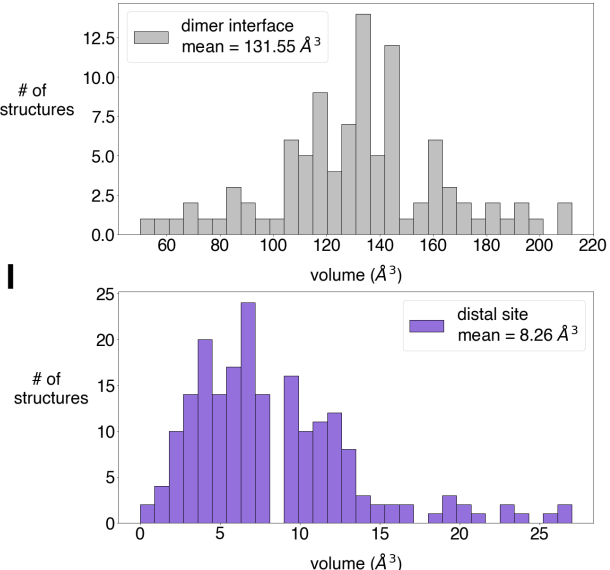

L

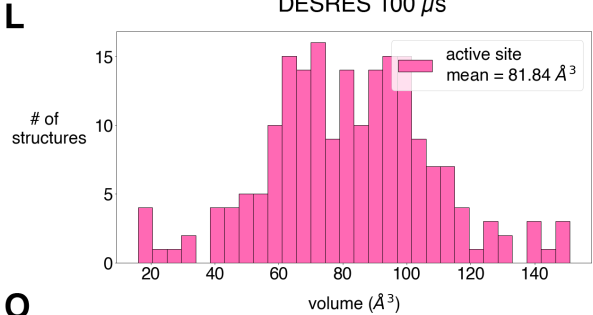

0
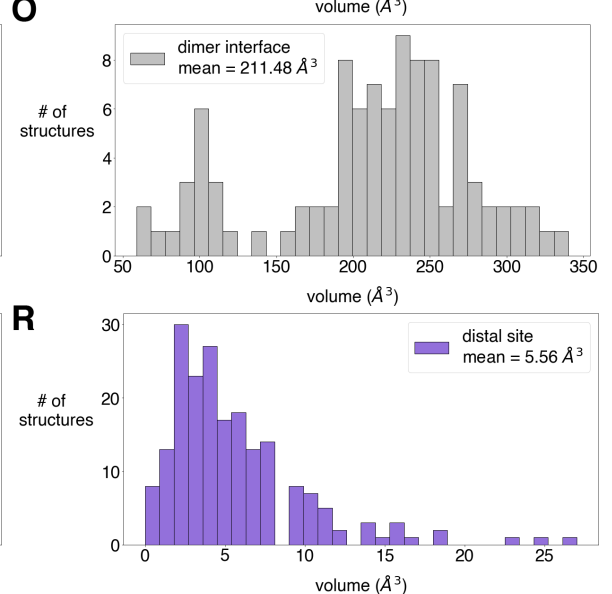

Figure S14 Pocket volumes calculated from GaMD versus brute force MD. (A,D,G) 200 ns GaMD. (B,E,H) 1 $\mu \mathrm{s}$ aggregate from five replicates of $200 \mathrm{~ns}$ GaMD. (C,F,I) $1 \mu \mathrm{s}$ of continuous GaMD. (J,M,P) $200 \mathrm{~ns}$ conventional MD. (K,N,Q) $10 \mu$ s of conventional MD carried out by Riken. (L,O,R) $100 \mu$ s of conventional MD carried out by D. E. Shaw Research (DESRES). 
A
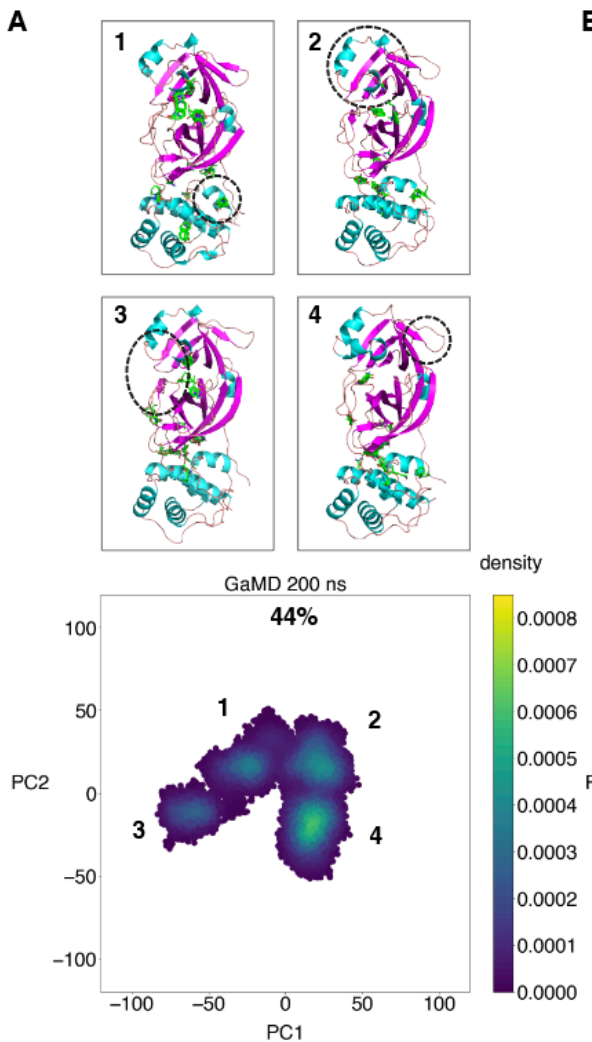

D
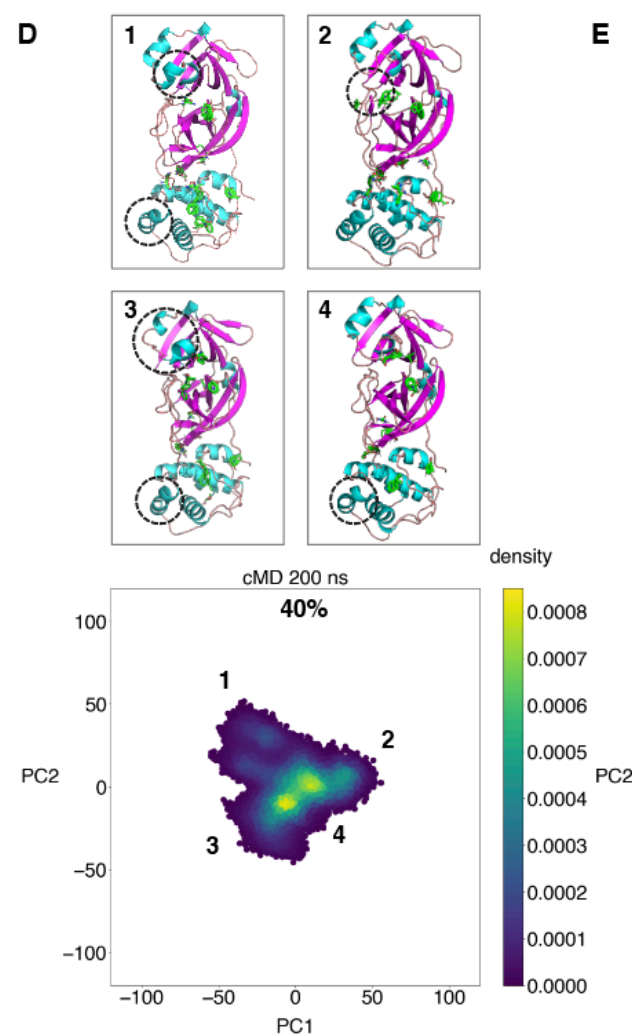

B
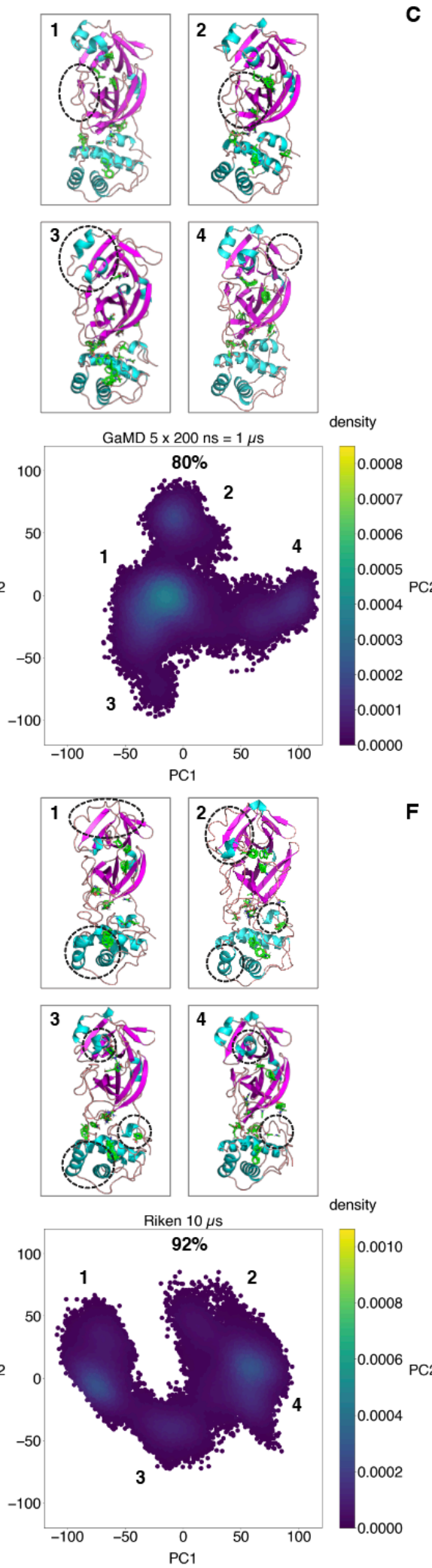

C


$\mathbf{F}$
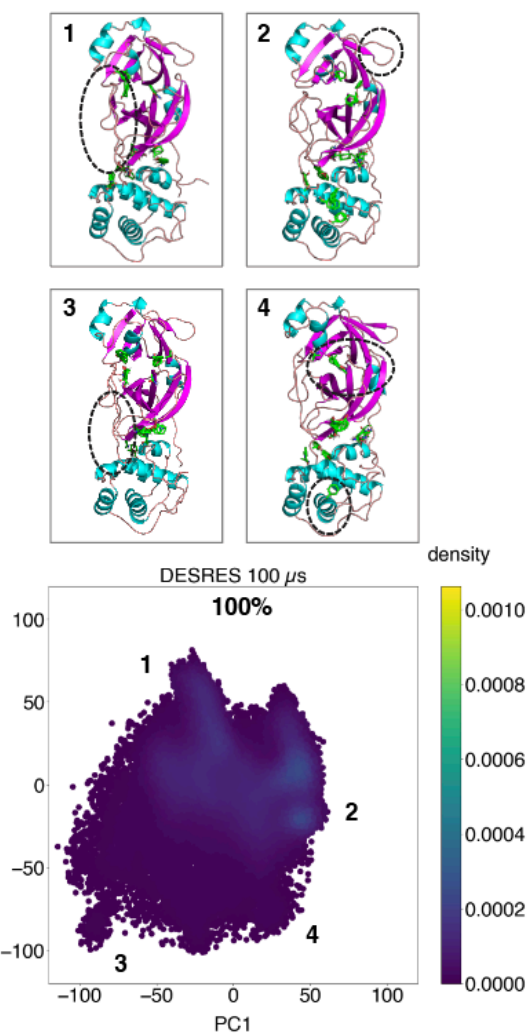

Figure S15 Structural comparison of conformations sampled with conventional MD and GaMD. Four structures from the most dense regions on the graphs from Figure $\mathbf{4}$ are labeled. Only chain A is shown for clarity, colored by secondary structure with blue helices, magenta beta sheets, and pink loops. Molecules from FTMap computational solvent mapping to structure are shown in green. Regions which noticeably differ from the crystal structure are outlined in dashed black lines. 


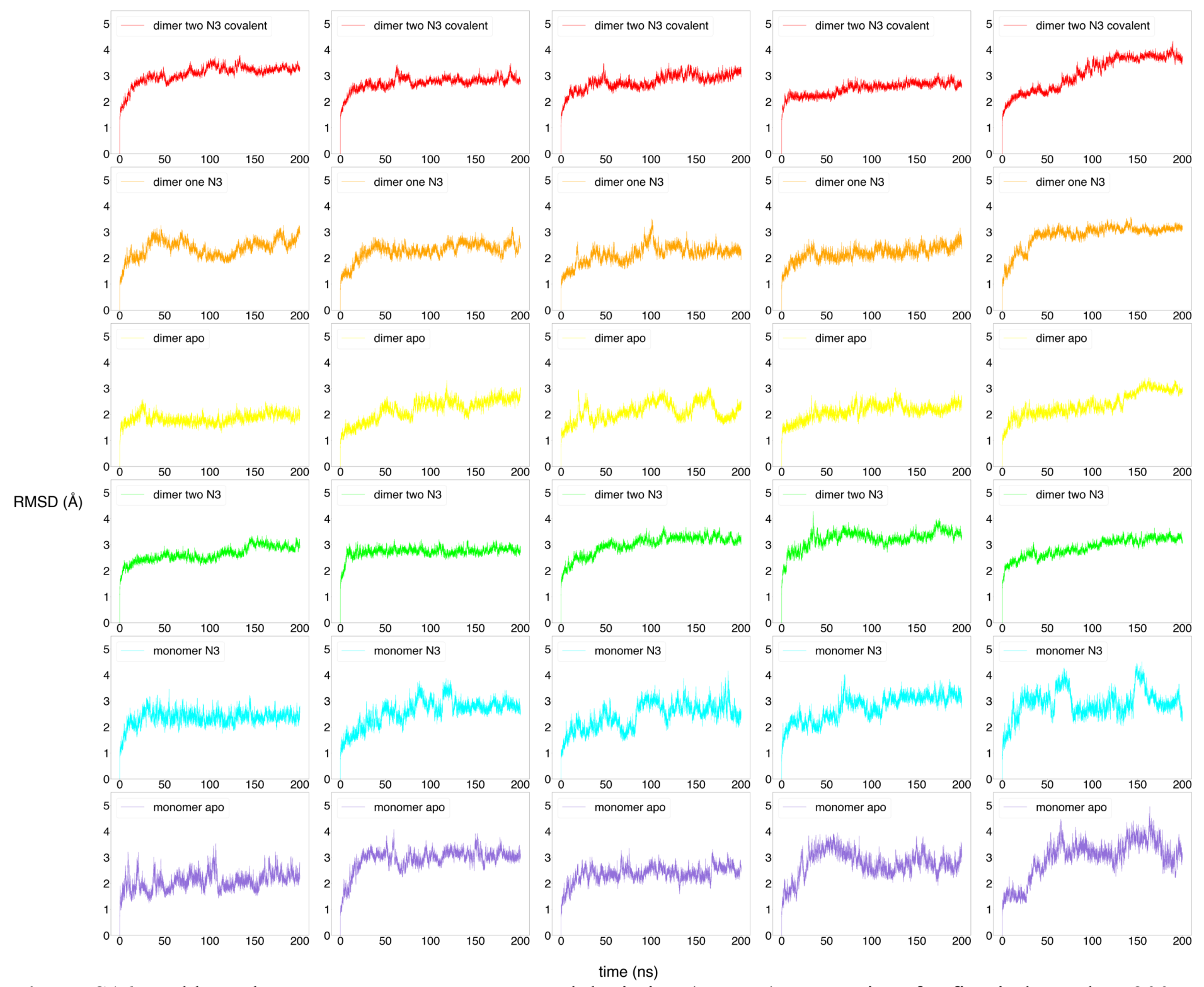

Figure S16 Backbone heavy-atom root mean squared deviation (RMSD) versus time for five independent 200 ns GaMD simulations, $1 \mu$ s aggregate simulation, for each of the six systems simulated as outlined in Figure S1. 


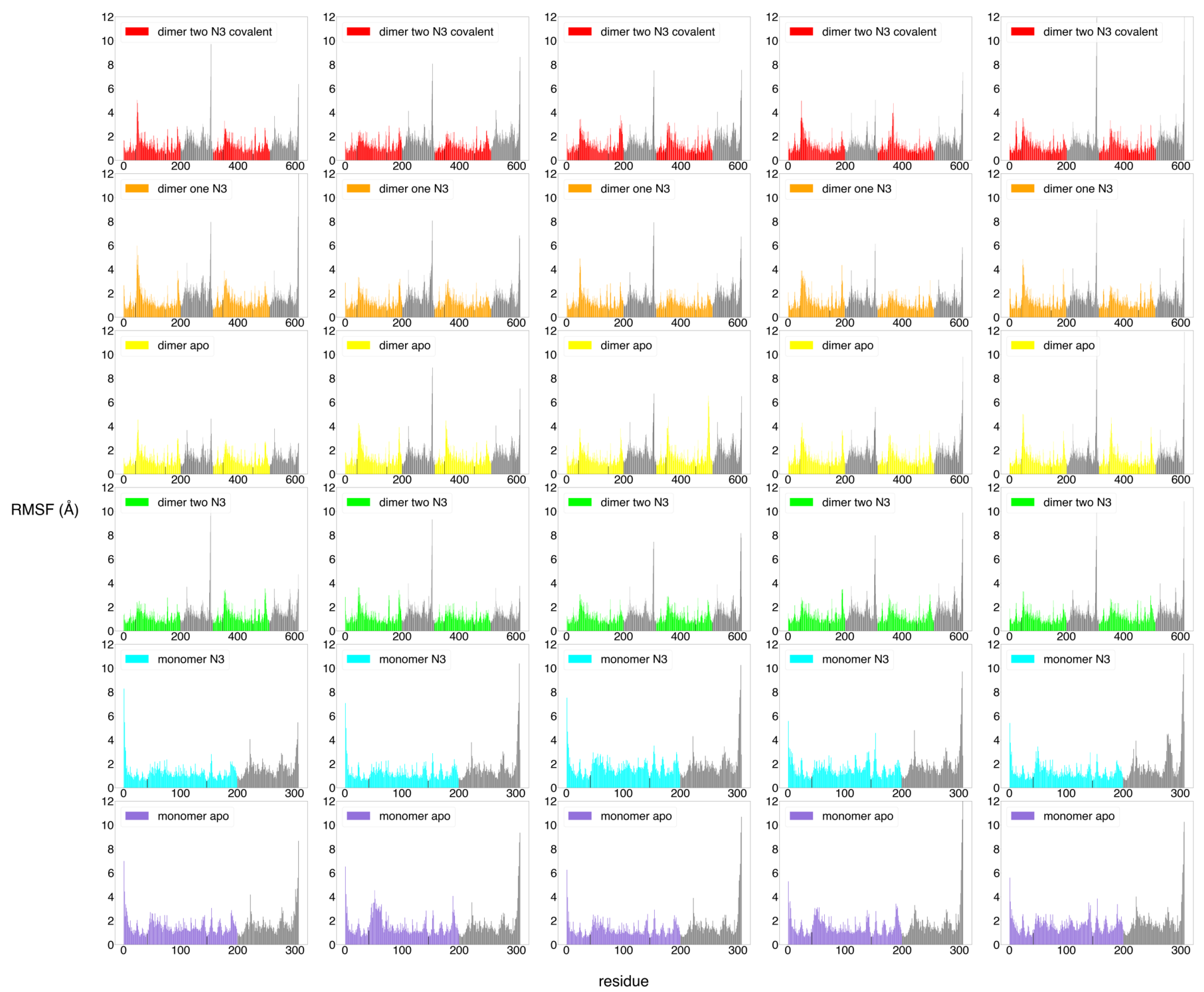

Figure S17 Root mean squared fluctuation of each residue, averaged across the simulation time, for each of the six systems outlined in Figure S1. C-terminal residues, comprising the distal region are colored grey, while the $\mathrm{N}$-terminal residues, comprising the active site region, are shown in color. Active site resides H41 and C145 are colored black. 


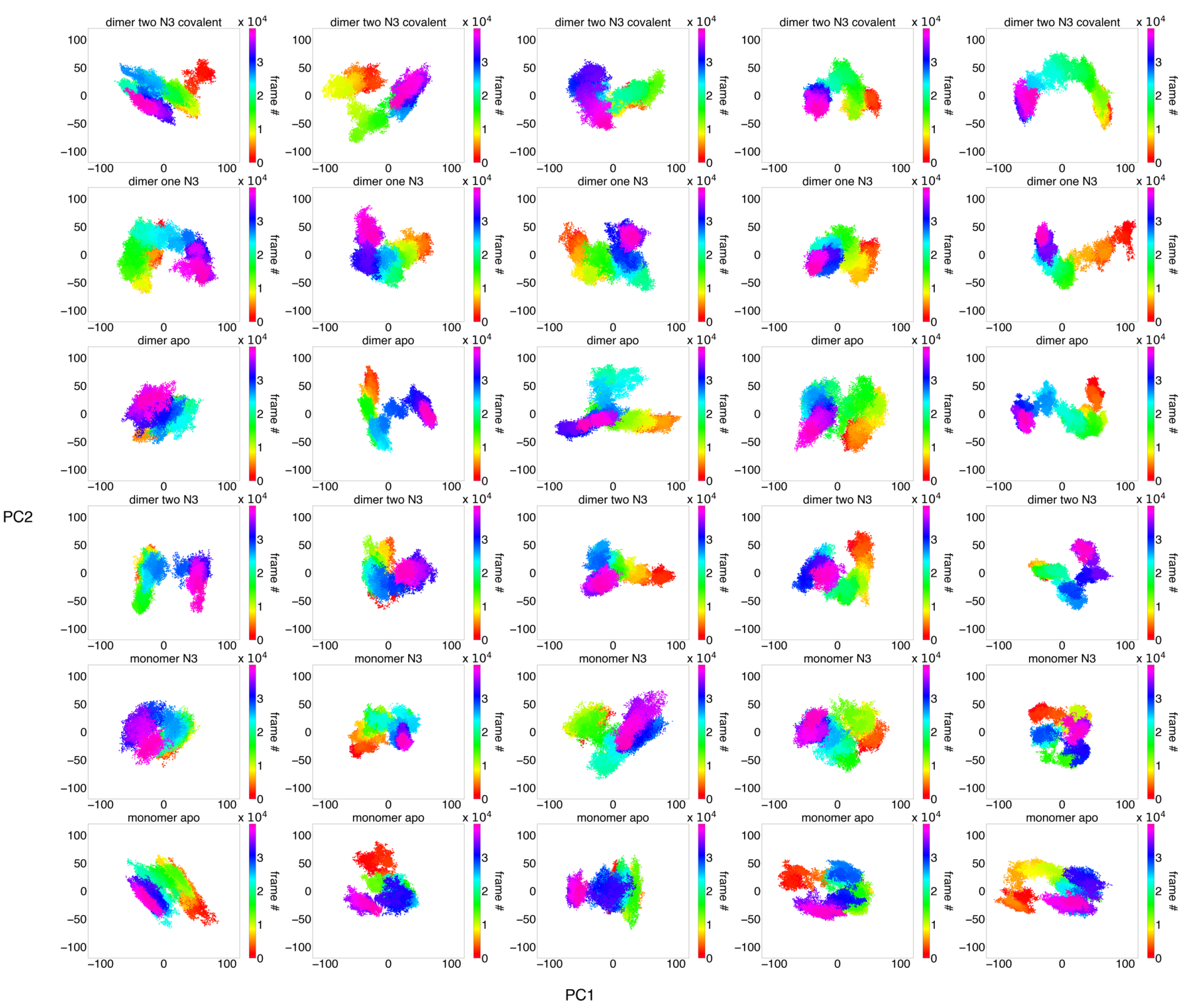

Figure S18 Plots of first and second principal components, projected from a mass weighted covariance matrix of backbone atom positions throughout the simulation for each of the six systems outlined in Figure S1. 


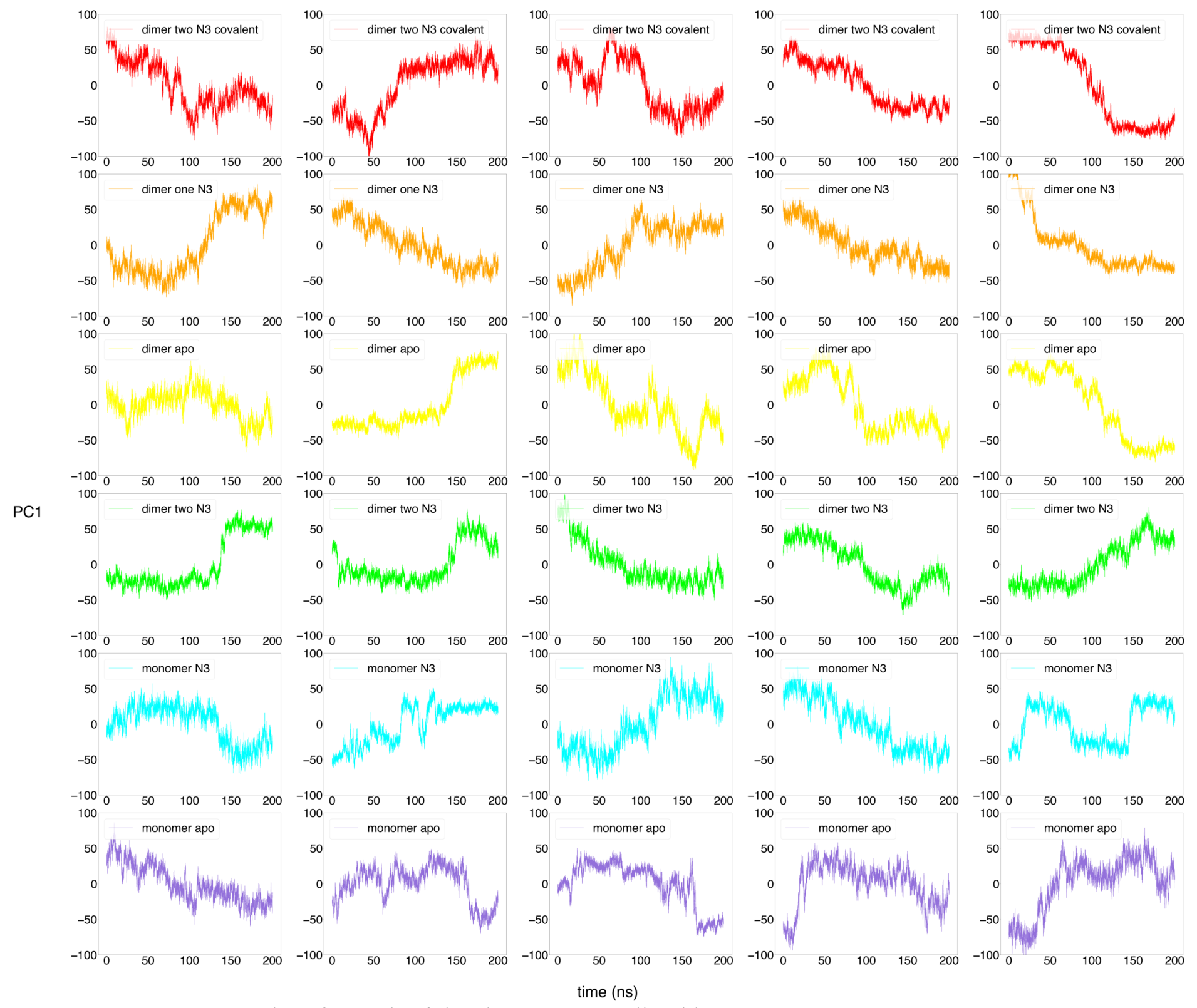

Figure S19 PC1 versus time for each of the six systems outlined in Figure S1. 


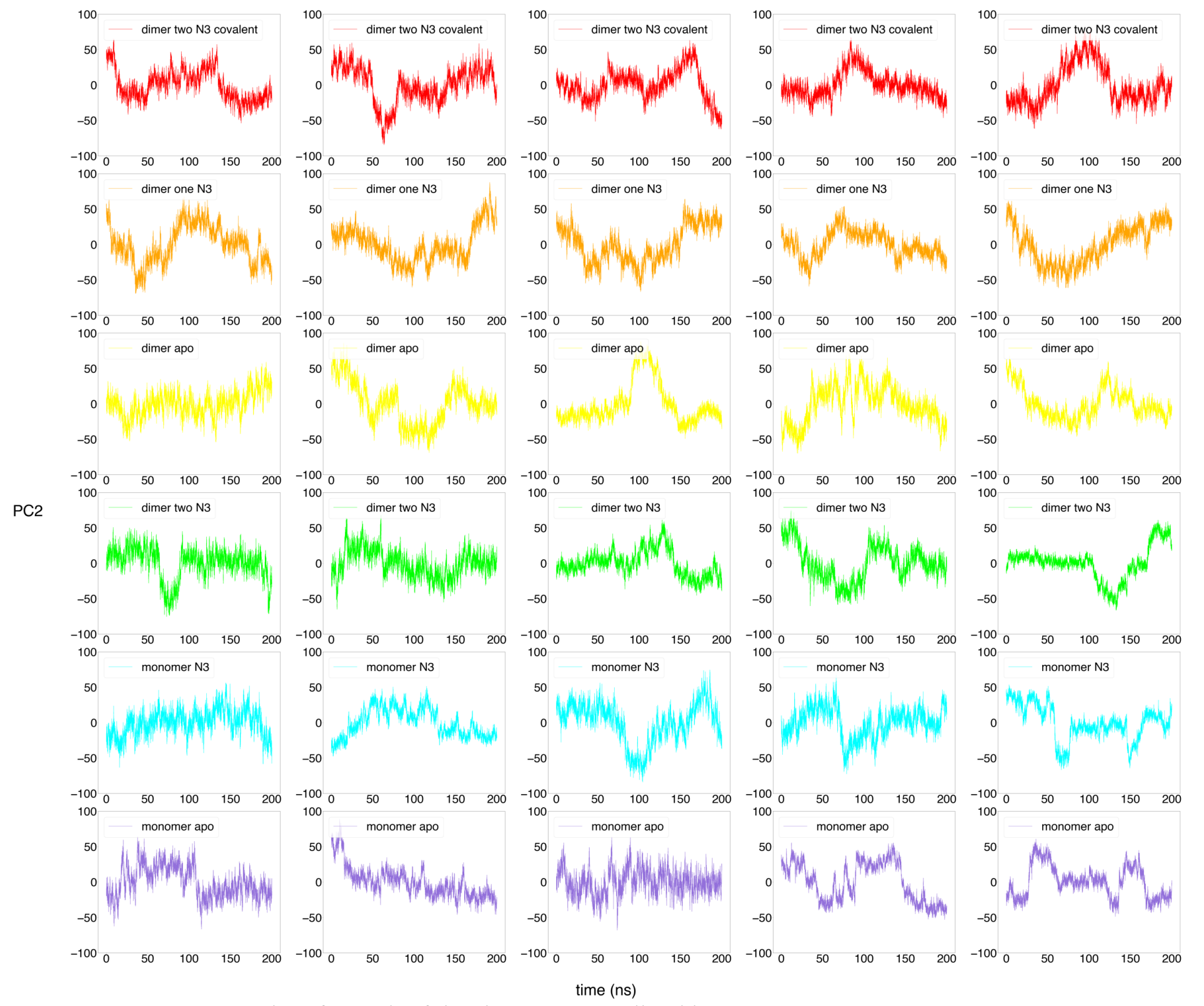

Figure S20 PC2 versus time for each of the six systems outlined in Figure S1. 


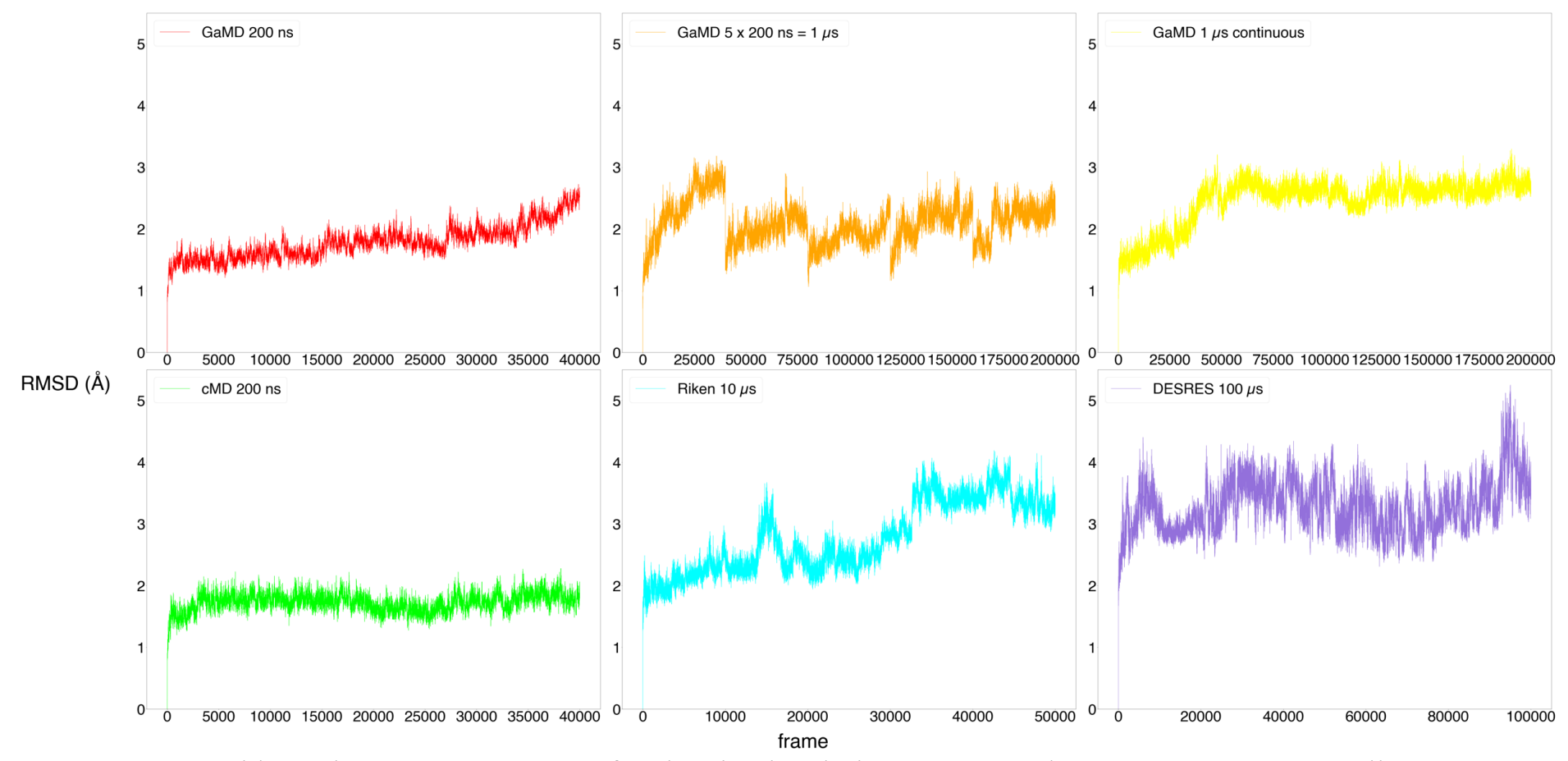

Figure S21 Backbone heavy-atom RMSD for the six simulations compared to assess GaMD sampling enhancement. Note that the GaMD 5 x 200ns simulation is composed of 5 concatenated trajectories, hence the apparent periodicity. 


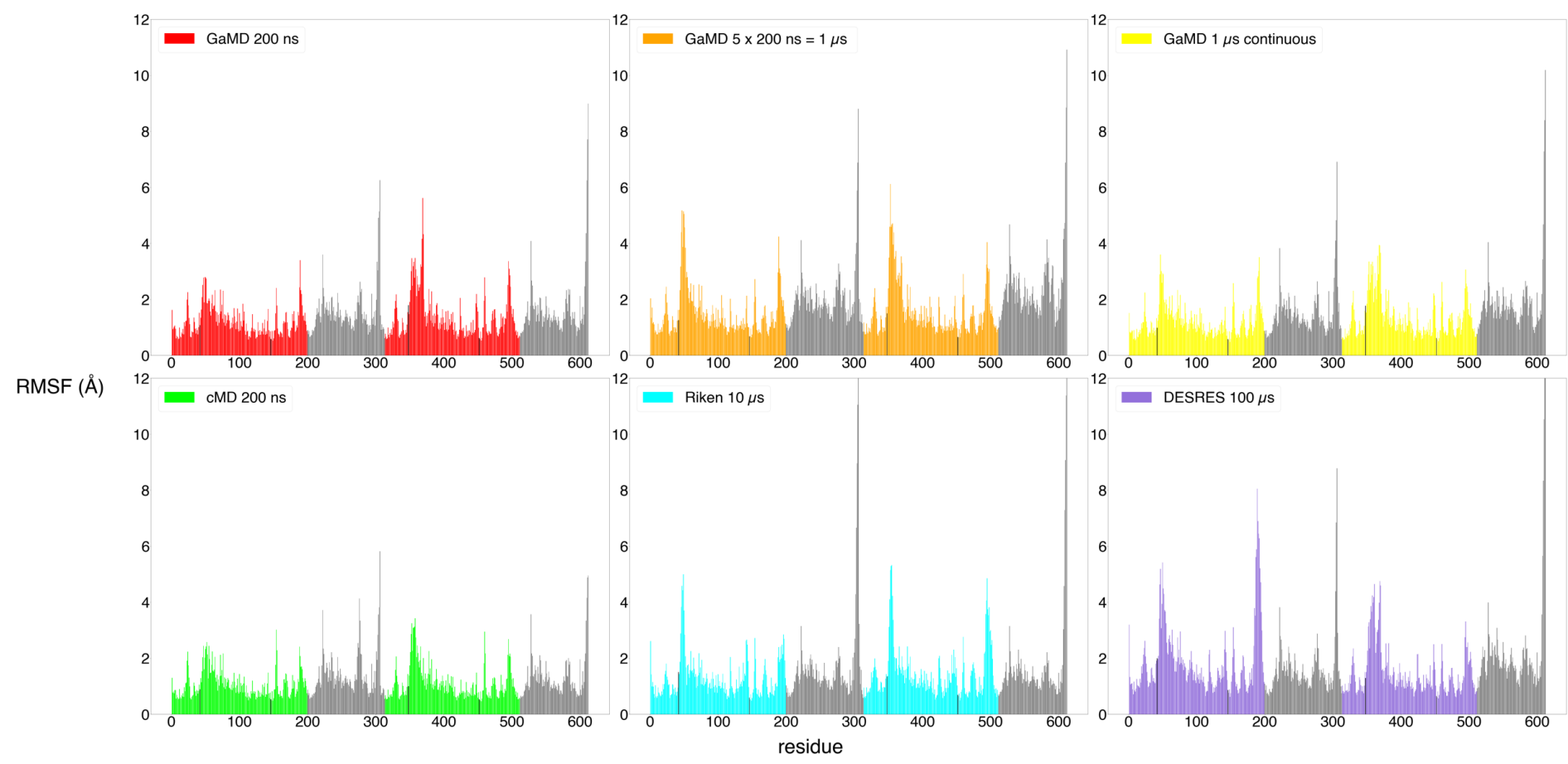

Figure S22 Root mean squared fluctuation of each residue, averaged across the simulation time, for the six simulations compared to assess GaMD sampling enhancement. C-terminal residues, comprising the distal region are colored grey, while the $\mathrm{N}$-terminal residues, comprising the active site region, are shown in color. Active site resides H41 and C145 are colored black. 
A

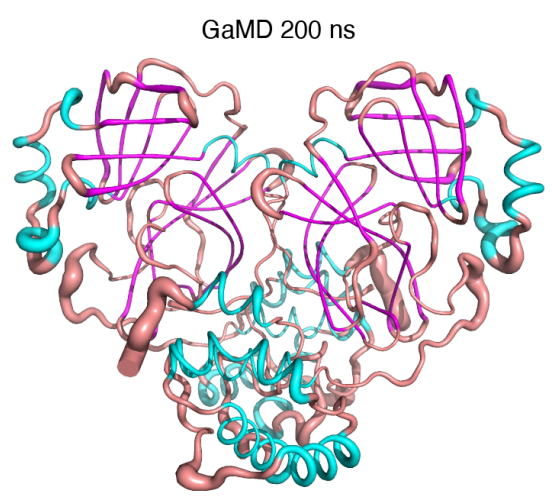

D

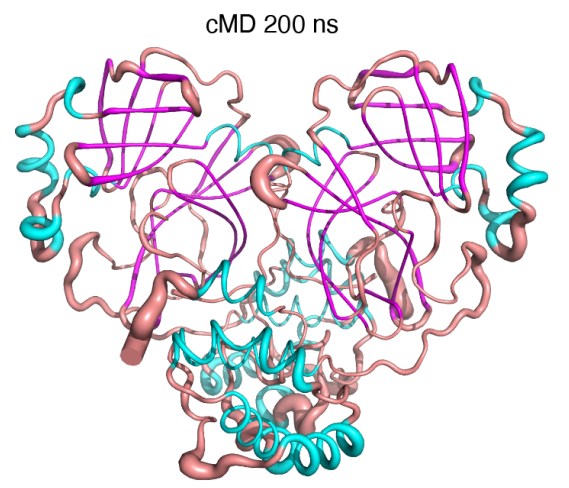

B

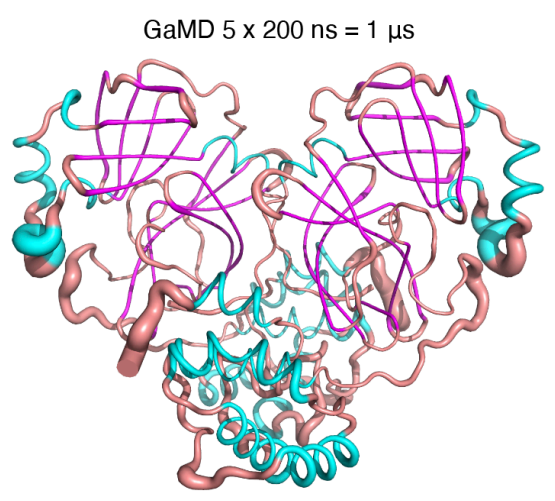

E

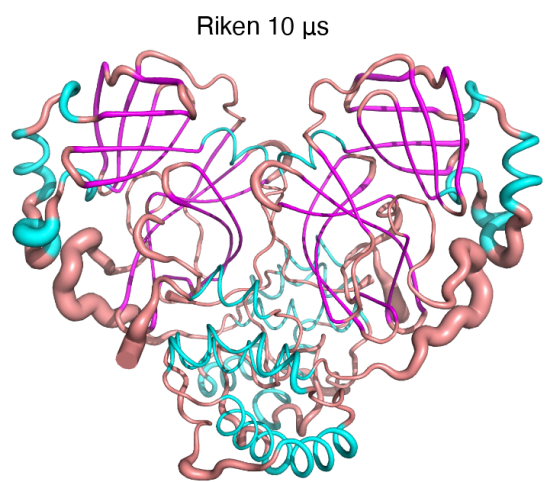

C

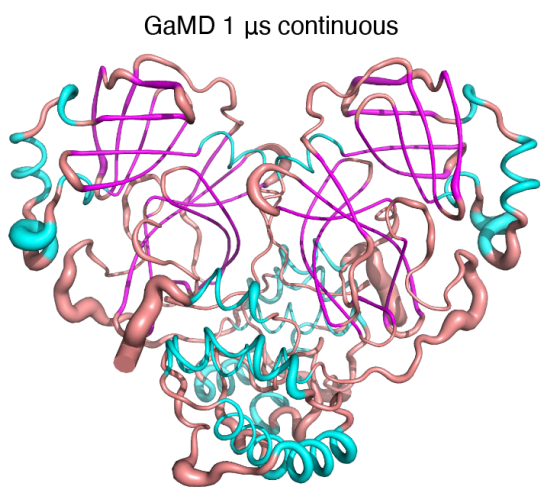

$\mathbf{F}$

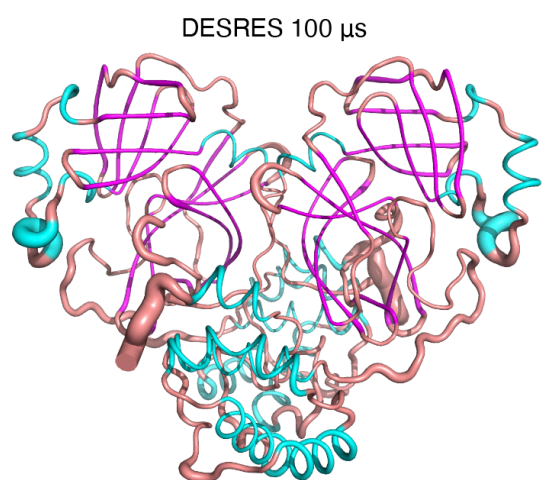

Figure S23 Visual representation of average RMSF comparing cMD and GaMD of different simulation lengths. The thickness of the cartoon is proportional to the magnitude of the RMSF, plotted on the apo dimeric form of 6LU7, colored by secondary structure. Helices are colored cyan, beta sheets are colored purple, and loops are colored pink. 


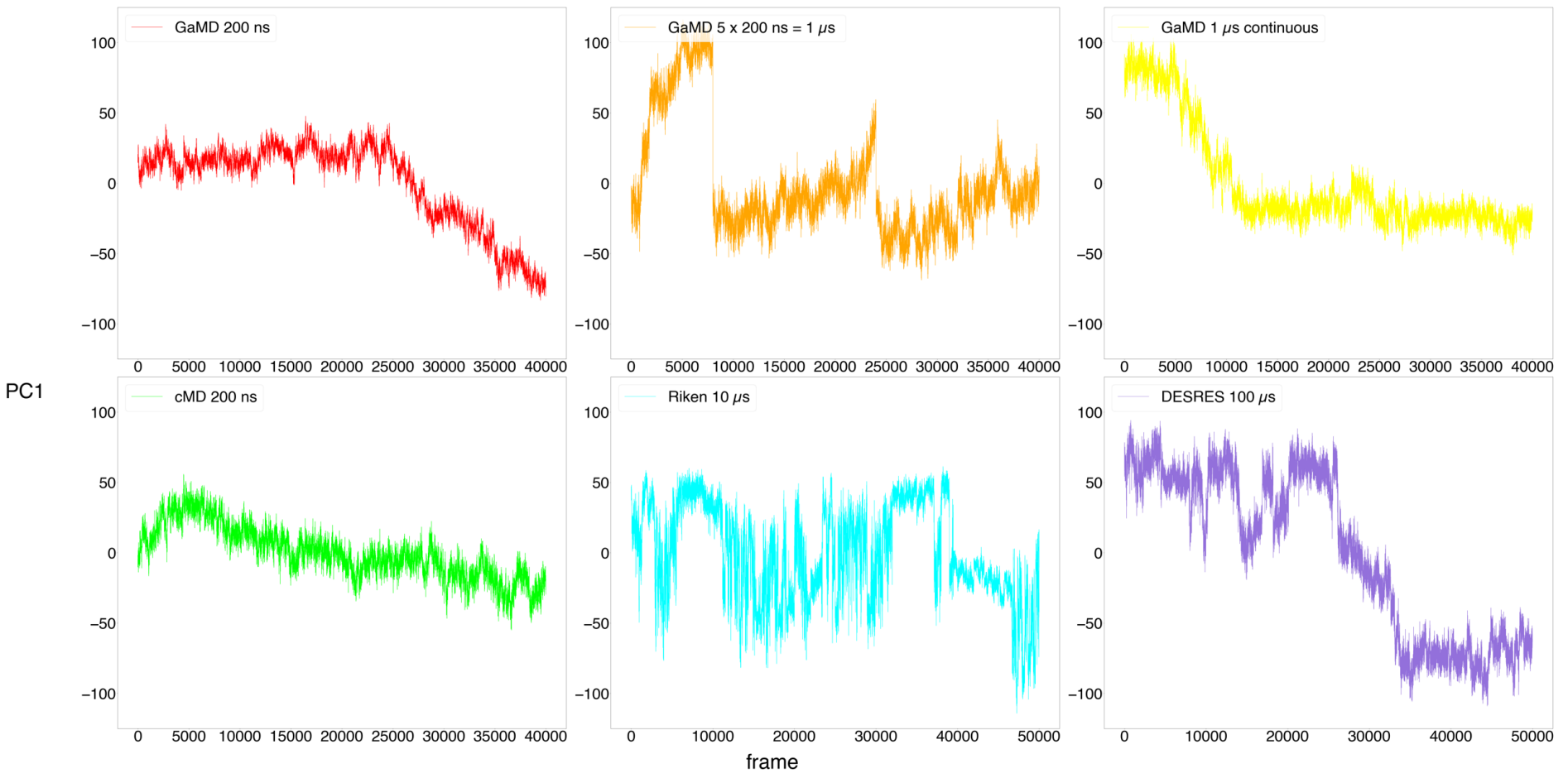

Figure S24 PC1 versus time for the six simulations compared to assess GaMD sampling enhancement. 


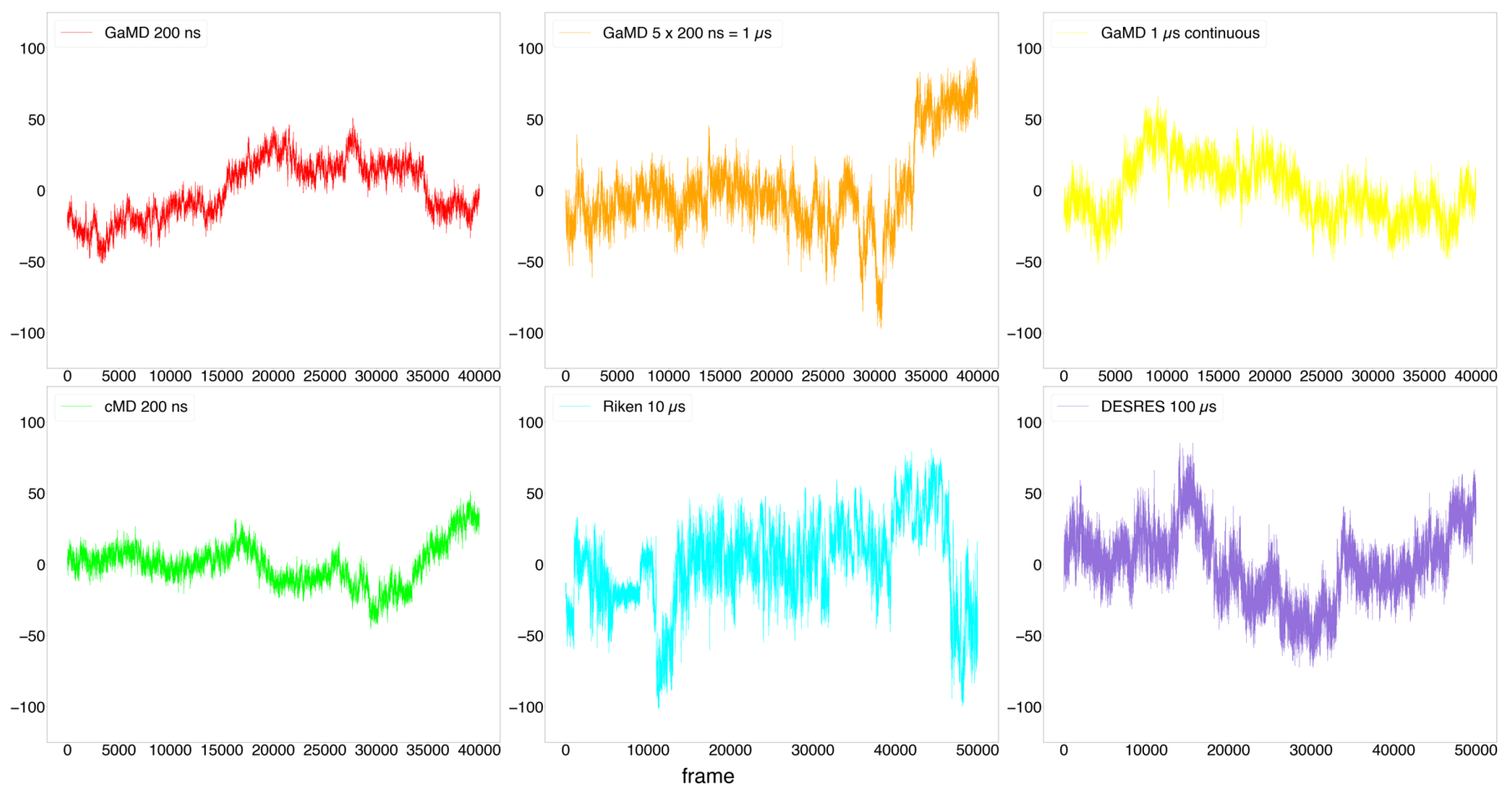

Figure S25 PC2 versus time for the six simulations compared to assess GaMD sampling enhancement. 


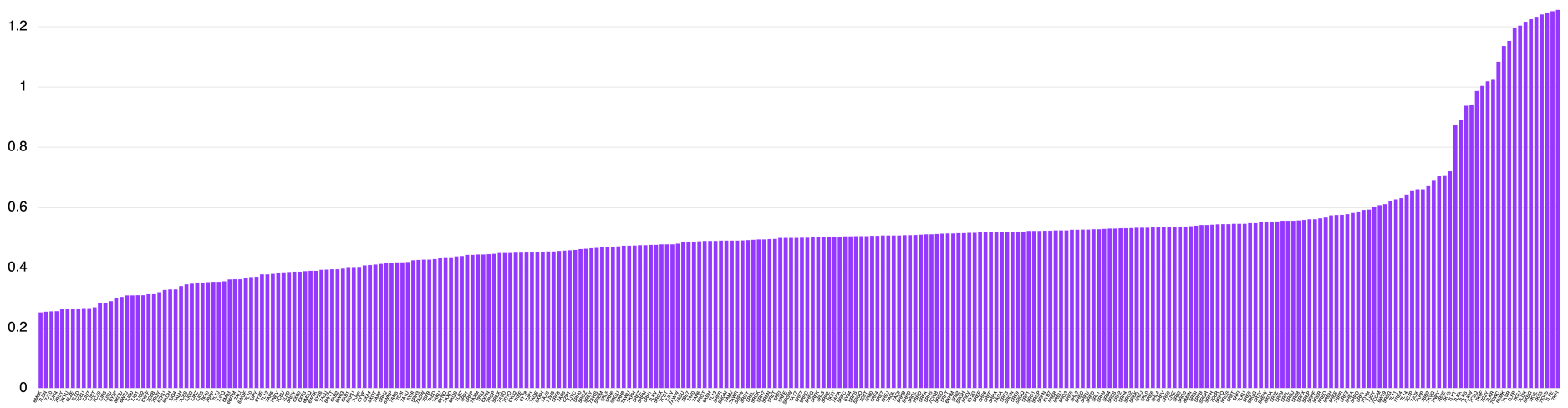

Figure S26 RMSD of 275 of $\mathrm{M}^{\text {pro }}$ crystal structures compared to PDB 6LU7. Structures were aligned in PyMOL and values obtained for each PDB code are listed in Data S1. 
Movie S1 Active site pocket dynamics sampled with GaMD. Trajectory from 200 ns simulation of the one N3 non-covalently bound $\mathrm{M}^{\text {pro }}$ dimer is shown, zoomed into the apo active site pocket. Catalytic dyad H41 and C145 are shown with sticks, dynamic loops as shown in Figure 2 are colored by residue, and a transparent surface of the pocket is shown with residues within $6 \AA$ of $\mathrm{H} 41$.

Data S1 Values for RMSD of 275 of $\mathrm{M}^{\text {pro }}$ crystal structures compared to PDB 6LU7 